\title{
Uncertainties in field-line tracing in the magnetosphere. Part II: the complete internal geomagnetic field
}

\author{
D. M. Willis ${ }^{1, *}$, J. Robin Singh ${ }^{1,2}$, K. S. C. Freeman ${ }^{1}$ \\ ${ }^{1}$ Rutherford Appleton Laboratory, Chilton, Didcot, Oxon OX11 0QX, UK \\ ${ }^{2}$ School of Mathematical and Information Sciences, Coventry University, Coventry, CV1 5FB, UK \\ Received: 6 November 1995 / Revised: 30 May 1996 / Accepted: 11 September 1996
}

\begin{abstract}
The discussion in the preceding paper is restricted to the uncertainties in magnetic-field-line tracing in the magnetosphere resulting from published standard errors in the spherical harmonic coefficients that define the axisymmetric part of the internal geomagnetic field (i.e. $g_{n}^{0} \pm \delta g_{n}^{0}$ ). Numerical estimates of these uncertainties based on an analytic equation for axisymmetric field lines are in excellent agreement with independent computational estimates based on stepwise numerical integration along magnetic field lines. This comparison confirms the accuracy of the computer program used in the present paper to estimate the uncertainties in magnetic-field-line tracing that arise from published standard errors in the full set of spherical harmonic coefficients, which define the complete (non-axisymmetric) internal geomagnetic field (i.e. $g_{n}^{m} \pm \delta g_{n}^{m}$ and $h_{n}^{m} \pm \delta h_{n}^{m}$ ). An algorithm is formulated that greatly reduces the computing time required to estimate these uncertainties in magnetic-field-line tracing. The validity of this algorithm is checked numerically for both the axisymmetric part of the internal geomagnetic field in the general case $(1 \leq n \leq 10)$ and the complete internal geomagnetic field in a restrictive case $(0 \leq m \leq n, 1 \leq n \leq 3)$. On this basis it is assumed that the algorithm can be used with confidence in those cases for which the computing time would otherwise be prohibitively long. For the complete internal geomagnetic field, the maximum characteristic uncertainty in the geocentric distance of a field line that crosses the geomagnetic equator at a nominal dipolar distance of 2 $R_{\mathrm{E}}$ is typically $100 \mathrm{~km}$. The corresponding characteristic uncertainty for a field line that crosses the geomagnetic equator at a nominal dipolar distance of $6 R_{\mathrm{E}}$ is typically $500 \mathrm{~km}$. Histograms and scatter plots showing the characteristic uncertainties associated with magneticfield-line tracing in the magnetosphere are presented for
\end{abstract}

\footnotetext{
* Also Visiting Reader in Physics, University of Sussex, Falmer, Brighton, BN1 9QH, UK

Correspondence to: D. M. Willis
}

a range of illustrative examples. Finally, estimates are given for the maximum uncertainties in the locations of the conjugate points of selected geophysical observatories. Numerical estimates of the uncertainties in magnetic-field-line tracing in the magnetosphere, including the associated uncertainties in the locations of the conjugate points of geophysical observatories, should be regarded as "first approximations" in the sense that these estimates are only as accurate as the published standard errors in the full set of spherical harmonic coefficients. As in the preceding paper, however, all computational techniques developed in this paper can be used to derive more realistic estimates of the uncertainties in magnetic-field-line tracing in the magnetosphere, following further progress in the determination of more accurate standard errors in the spherical harmonic coefficients.

\section{Introduction}

As noted in the introduction to the preceding paper by Willis, Singh, and Comer (1997), hereafter referred to as Paper I, the technique of tracing along magnetic field lines is widely used in magnetospheric physics to provide a "magnetic frame of reference" that facilitates both the planning of experiments and the interpretation of observations. The importance of developing realistic representations of both the Earth's internal magnetic field (i.e. DGRF or IGRF) and the total magnetic field in the magnetosphere is emphasized in Paper I, and it is not necessary to repeat that discussion here. It suffices to note again that an accurate model of the magnetic field in the magnetosphere would be of great value in solarterrestrial physics, particularly in comparisons between measurements made by ground-based and satelliteborne instruments. It should be stressed once more, however, that the present pair of papers begin a systematic study of the uncertainties in field-line tracing 
in the magnetosphere by considering first those uncertainties that arise solely from possible errors in the specification of the geomagnetic field of internal origin. It must be acknowledged that uncertainties in the specification of the external sources of magnetic field in the magnetosphere (viz. ionospheric, Birkeland, ring, magnetopause and magnetotail currents) may well produce larger uncertainties in field-line tracing, particularly for magnetic field lines emanating at high geomagnetic latitudes (i.e. those passing through the outer magnetosphere). Nevertheless, a detailed discussion of the uncertainties in field-line tracing for just the geomagnetic field of internal origin (DGRF or IGRF) represents a significant advance that has important applications and implications in several branches of solar-terrestrial physics.

The estimation of the uncertainties in magnetic-fieldline tracing in the magnetosphere for the geomagnetic field of internal origin is divided into two separate papers for the following reasons. As noted in Paper I, an exact analytic equation exists for the magnetic field lines of an arbitrary linear combination of axisymmetric multipoles (Backus, 1988). This equation is used in Paper I to derive accurate numerical estimates of the uncertainties in field-line tracing that are due to the standard errors in the axisymmetric spherical harmonic coefficients (i.e. $g_{n}^{0} \pm \delta g_{n}^{0}$ ) published by Langel et al. (1992). In addition, it is also possible to calculate these uncertainties by using a stepwise numerical integration procedure to trace along magnetic field lines. It is shown in Paper I that, for the axisymmetric part of the geomagnetic field, the uncertainties derived by stepwise numerical integration along magnetic field lines are essentially identical to those obtained independently using the exact equation due to Backus (1988). The excellent agreement between results derived by these two different techniques (cf. Tables 3 and 4 in Paper I) proves that the computer program used for stepwise numerical integration along magnetic field lines is very accurate. The primary purpose of this paper is to estimate the uncertainties in field-line tracing in the magnetosphere that are due to the standard errors in the full set of spherical harmonic coefficients (i.e. $g_{n}^{m} \pm \delta g_{n}^{m}, h_{n}^{m} \pm \delta h_{n}^{m}$ ) published by Langel et al. (1992). Since there is no known analytic equation for the magnetic field lines in the case of the complete (nonaxisymmetric) geomagnetic field of internal origin, uncertainties in field-line tracing in the magnetosphere must be calculated using stepwise numerical integration along magnetic field lines.

The main goals of this paper are fourfold: (i) provision of a concise specification and assessment of the computer program used for stepwise numerical integration along magnetic field lines; (ii) generation of an algorithm that drastically reduces the time required to compute uncertainties in field-line tracing for the complete internal geomagnetic field; (iii) presentation of idealized illustrative results for comparison with the corresponding results in Paper I; and (iv) calculation of uncertainties in the locations of conjugate points for selected geophysical observatories.

\section{The internal magnetic field of the Earth}

The geomagnetic field of internal origin is defined in this section. For completeness, the magnetic-field components $\left(B_{r}, B_{\theta}, B_{\phi}\right)$, which are defined implicitly by Eq. 1 of Paper I $(\boldsymbol{B}=-\operatorname{grad} V)$, are presented explicitly in Sect. 2.1. This approach admittedly involves some slight repetition in the definition of the terms that appear in the normal spherical harmonic expansion of the internal geomagnetic field, but it does help to make this paper largely self-contained. For the same reason, the concept of the "range" of a geomagnetic-field model, which is introduced in Sect. 2.4 of Paper I, is summarized succinctly in Sect. 2.2 of this paper. The general differential equations that define the magnetic field lines in the magnetosphere are presented in Sect. 2.3. Stepwise numerical integration along a magnetic field line is essentially equivalent to finding a numerical solution of these differential equations.

\subsection{The components of the internal magnetic field}

It follows from Eq. 1 of Paper I that at any given instant of time (epoch) the three magnetic-field components of the Earth's internal magnetic field can be expressed in the form $(\boldsymbol{B}=-\operatorname{grad} V)$

$$
\begin{aligned}
B_{r}= & \sum_{n=1}^{\infty}(n+1)\left(R_{\mathrm{E}} / r\right)^{n+2} \sum_{m=0}^{n}\left(g_{n}^{m} \cos m \phi+h_{n}^{m} \sin m \phi\right) \\
& \times P_{n}^{m}(\cos \theta), \\
B_{\theta}= & -\sum_{n=1}^{\infty}\left(R_{\mathrm{E}} / r\right)^{n+2} \sum_{m=0}^{n}\left(g_{n}^{m} \cos m \phi+h_{n}^{m} \sin m \phi\right) \\
& \times d P_{n}^{m}(\cos \theta) / d \theta \\
B_{\phi}= & \sum_{n=1}^{\infty}\left(R_{\mathrm{E}} / r\right)^{n+2} \sum_{m=0}^{n} m\left(g_{n}^{m} \sin m \phi-h_{n}^{m} \cos m \phi\right) \\
& \times P_{n}^{m}(\cos \theta) / \sin \theta .
\end{aligned}
$$

These magnetic-field components are valid only outside the region of origin of the Earth's internal magnetic field (predominantly the liquid metallic outer core), in an ideal external region containing no sources of magnetic field (i.e. curl $\boldsymbol{B}=0$ ). Positions on the surface of the Earth are specified in terms of geocentric spherical polar coordinates $(r, \theta, \phi)$ with origin $\mathrm{O}$ at the centre of the Earth; $r$ is the geocentric radial distance in $\mathrm{km}\left(r \geq R_{\mathrm{E}}\right)$, $\theta$ is the geographic co-latitude with the north geographic pole at $\theta=0$, and $\phi$ is the geographic longitude measured east from Greenwich. The radius of the reference sphere, $r=R_{\mathrm{E}}$, is taken to be the mean radius of the Earth $(6371.2 \mathrm{~km}) ; P_{n}^{m}(\cos \theta)$ is Schmidt's partially (or quasi-) normalized associated Legendre function of order $m$ and degree $n$ (where $m$ and $n$ are integers; $m \in \mathbb{I}_{0}^{+}$and $\left.n \in \mathbb{I}^{+}\right) ; g_{n}^{m}$ and $h_{n}^{m}$ are the spherical harmonic (or Schmidt) coefficients for the particular epoch considered, expressed in units of nanotesla (nT); and all physical quantities are measured in SI units. In 
this paper the definition of $\boldsymbol{B}$ is such that the spherical harmonic coefficients $g_{n}^{m}$ and $h_{n}^{m}$ have the (conventional) dimensions of magnetic induction.

\subsection{Range of a geomagnetic-field model}

As in Sect. 2.4 of Paper I, each spherical harmonic coefficient $g_{n}^{m}$ is assumed to lie in the interval of real numbers $\left[g_{n}^{m}-\delta g_{n}^{m}, g_{n}^{m}+\delta g_{n}^{m}\right]$, which is represented by the ordered pair of real numbers (the "lower" and "upper" end-points), $g_{n}^{m}-\delta g_{n}^{m}$ and $g_{n}^{m}+\delta g_{n}^{m}\left(\delta g_{n}^{m} \geq 0\right)$; similarly for $h_{n}^{m}$. The "range", or variability, of a geomagnetic-field model (DGRF or IGRF) at any epoch $t_{0}$ is defined in terms of the set of "limiting geomagnetic-field models", which results from selecting every possible permutation of the pairs of "end-points" for $g_{n}^{m}$ and $h_{n}^{m}$. If the infinite summations in Eqs. 1-3 are truncated at degree $n_{\max }$, the number $(N)$ of spherical harmonic coefficients is given by $N=n_{\max }\left(n_{\max }+2\right)$. The number $(\mathscr{N})$ of "limiting geomagnetic-field models" $(\mathscr{G})$ is then given by $\mathscr{N}=2^{N}$ and the set of such field models can be expressed symbolically in the form $\left\{\mathscr{G}_{1}, \mathscr{G}_{2}, \mathscr{G}_{3}, \cdots, \mathscr{G}_{\mathcal{N}}\right\}$. Since this variability must be referred to the mean (or central) geomagnetic-field model $\mathscr{G}_{0}$ (which is defined by the set of central values $g_{n}^{m}$ and $\left.h_{n}^{m}\right)$, the number of different "models" to be considered in this method of determining the uncertainty in field-line tracing is $\mathscr{N}+1$, that is $2^{N}+1$.

The uncertainty in the geocentric distance $(r)$ of an arbitrary point on a magnetic field line is denoted symbolically by $\delta r$, where $\delta r \in \mathbb{R}_{0}^{+}$. The mean (or central) model $\mathscr{G}_{0}$ corresponds to $r$, whereas the models with errors associated with them, namely $\left\{\mathscr{G}_{1}, \mathscr{G}_{2}, \mathscr{G}_{3}, \cdots, \mathscr{G}_{\mathcal{N}}\right\}$, correspond conceptually to a set of values $\{r+\delta r\}$. However, in the case of the complete internal geomagnetic field $(m \neq 0)$, the set of values $\{r+\delta r\}$ actually defines a small surface area, rather than a linear (or curvilinear) interval, as occurs in the case of the axisymmetric part of the internal geomagnetic field (cf. Sect. 2.4 in Paper I). Therefore, $\delta r$ and $r$ should really be regarded as vectors in the sense that $\boldsymbol{\delta} \boldsymbol{r}$ has both a magnitude $\delta r(|\boldsymbol{\delta} \boldsymbol{r}|)$ and an azimuthal angle with respect to the central vector $\boldsymbol{r}$. Although this definition of $\boldsymbol{\delta} \boldsymbol{r}$ is slightly imprecise, it is clear in principle that $\delta \boldsymbol{r}$ can be measured uniquely with respect to $\boldsymbol{r}$ in any convenient plane, or on any other welldefined surface, that passes through the point defined by $\boldsymbol{r}$. As in Paper I, an important goal is to find the largest possible value for $\delta r\left(\delta r_{\max }\right)$ at the geomagnetic equator, or on the surface of the Earth in the opposite hemisphere, for any given starting point on the surface of the Earth.

Reference to Table 1 of Paper I shows that the number of "limiting geomagnetic-field models" exceeds $3.4 \times 10^{10}$ even in the restrictive case $n_{\max }=5$. The purpose of this paper is to consider the more realistic case $n_{\max }=10$, which corresponds to more than $1.3 \times 10^{36}$ "limiting geomagnetic-field models" for the complete geomagnetic field of internal origin (DGRF or IGRF). In principle, the determination of $\delta r_{\max }$ involves stepwise numerical integration along a magnetic field line for every limiting geomagnetic-field model. In practice, the computing time required to estimate uncertainties in field-line tracing in the magnetosphere by this technique can be prohibitively long. An indication of the computing times required to calculate $\delta r_{\max }$ by the various methods considered in Paper I $\left[N=n_{\max }\right]$ and this paper $\left[N=n_{\max }\left(n_{\max }+2\right)\right]$ is given in Appendix A. All computations have been performed using a DEC 3000 AXP (Alpha) - Model 400 computer. Also indicated in Appendix A are those cases that have been considered $(\checkmark)$ and those cases not considered $(\boldsymbol{X})$ simply because the computing time is inordinately long. An algorithm that drastically reduces the computing time required to calculate $\delta r_{\max }$ is formulated in Sect. 4.

\subsection{Differential equations for the magnetic field lines}

The universal differential equations for the magnetic field lines of a general magnetic field $\left(B_{r}, B_{\theta}, B_{\phi}\right)$ are expressible as follows

$\frac{d r}{B_{r}}=\frac{r d \theta}{B_{\theta}}=\frac{r \sin \theta d \phi}{B_{\phi}}=\frac{d s}{B}$,

where $d s$ denotes an element of arc length along a magnetic field line and $B=\left(B_{r}^{2}+B_{\theta}^{2}+B_{\phi}^{2}\right)^{1 / 2}$. In the present application to the internal geomagnetic field, the magnetic-field components $B_{r}, B_{\theta}$ and $B_{\phi}$ are defined by Eqs. 1, 2 and 3, respectively. For the axisymmetric part $(m=0)$ of the internal geomagnetic field, Eq. 4 simplifies to the single equation

$\frac{d r}{B_{r}}=\frac{r d \theta}{B_{\theta}}$,

where $B_{r}$ and $B_{\theta}$ are defined by Eqs. 1 and 2 with $m=0$. Note from Eq. 3 that $B_{\phi} \equiv 0$ if $m=0$, which is a necessary and sufficient condition for an axisymmetric magnetic field.

An exact equation for the magnetic field lines of an individual axisymmetric magnetic multipole of arbitrary degree $(n)$ has been derived by Willis and Young (1987), who solved Eq. 5 for the appropriate magnetic-field components. In this special case, the magnetic-field components can be obtained from Eqs. 1 and 2 by putting $m=0$, taking just the $n^{\text {th }}$ terms in the summations for both $B_{r}$ and $B_{\theta}$, and using the relation $d P_{n}^{0}(\cos \theta) / d \theta=-[n(n+1) / 2]^{1 / 2} P_{n}^{1}(\cos \theta)$. The equation derived by Willis and Young (1987) is presented as Eq. 6 in Paper I and this reduces correctly to the wellknown equation for the field lines of a magnetic dipole if $n=1$ (cf. Sect. 3.1 of Paper I). Subsequently, Jeffreys (1988) presented an alternative, and somewhat simpler, mathematical derivation of the equation for the field lines of a single axisymmetric multipole and Backus (1988) generalized this result to the case of an arbitrary linear combination of axisymmetric multipoles. Backus did not solve Eq. 5 explicitly, but his solution, which is presented as Eq. 4 in Paper I, obviously satisfies Eq. 5 
here. The analytic equation derived by Backus is used in Paper I to estimate the uncertainties in magnetic-fieldline tracing for the axisymmetric part $(m=0)$ of the internal geomagnetic field and to check the accuracy of the computer program used in this paper to estimate the corresponding uncertainties in field-line tracing for the complete internal geomagnetic field. By solving the three-dimensional Eq. 4 above, Willis and Gardiner (1988) derived exact equations for the magnetic field lines of two special non-axisymmetric magnetic multipoles of arbitrary degree $(n)$, which may be classified as either symmetric $(m=n)$ or antisymmetric $(m=n-1)$ sectorial multipoles. Analytic solutions to Eq. 4 are relatively rare, however, and it is usually necessary to solve these equations by numerical techniques. The computer program used to solve Eq. 4 by stepwise numerical integration along magnetic field lines in the case of the complete internal geomagnetic field is discussed in the following section.

\section{The computer program used to trace along magnetic field lines}

The provenance of the computer program used to trace along magnetic field lines by stepwise numerical integration is uncertain. In the absence of complete bibliographic references, which would accord proper recognition to the original authors, an attempt is made to note very briefly the origins of the program and to explain clearly the procedures employed. Although the primary purpose in this section is to discuss the use of the computer program in determining uncertainties in field-line tracing in the magnetosphere, it is hoped that the following documentation of the program will help to clarify and validate its use in other contexts.

\subsection{The origin of the field-line tracing program}

The program was obtained from the EISCAT Scientific Association as part of a suite of programs and subroutines concerned with various aspects of the Earth's magnetic field. Unfortunately, the origin of some of these routines is now obscure. Although the package was based on a subroutine library from the Stanford Research Institute in California, it is not known if all the routines originated from that source. The program traces magnetic field lines by stepwise numerical integration from a given point on the surface of the Earth, or within the terrestrial magnetosphere, until a specified height or latitude is reached. The magnetic field originates solely from currents flowing in the Earth's liquid metallic outer core (i.e. DGRF or IGRF), and no account is taken of external currents flowing in the ionosphere and magnetosphere (apart from $\mathrm{S}_{\mathrm{q}}$ currents, as discussed in Sect. 2.3 of Paper I). This restriction is completely consistent with the assumption made throughout this study.

\subsection{The procedure for tracing along a magnetic field line}

To trace along a magnetic field line, the computer program performs a stepwise numerical calculation based on the condition that the tangent at each point on the field line is parallel to the magnetic field at that same point. Equation 4, which expresses this condition in mathematical form for a general magnetic field $\left(B_{r}, B_{\theta}, B_{\phi}\right)$, can be rewritten in vector form as follows,

$d \boldsymbol{r} / d s=\boldsymbol{B} /|\boldsymbol{B}|=\boldsymbol{b}$,

where $\boldsymbol{r}$ is the position vector measured from some convenient point (in this case the centre of the Earth), $s$ is the arc length along the magnetic field line, $\boldsymbol{B}$ is the magnetic-field vector, and $\boldsymbol{b}$ is a unit vector parallel to the magnetic field line. Equation 6 is used to calculate the increment in position vector from $\boldsymbol{r}$ to $\boldsymbol{r}+\boldsymbol{d} \boldsymbol{r}$. Starting at some initial value of $\boldsymbol{r}$, this equation is used to determine the location of the next point on the field line, and the process is repeated until the desired length of field line has been traced. The procedure employed requires the values of $\boldsymbol{b}$ and $d s$ to be known, the latter being an increment of arc length along the field line. The unit vector $\boldsymbol{b}$ presents no problems as it can be calculated from $\boldsymbol{b}=\boldsymbol{B} /|\boldsymbol{B}|$ using a suitable magnetic field model, but $d s$ requires some assumption about the shape of the field line. The simplest assumption is that of a straight line in direction $\boldsymbol{b}$ between one point on the field line and the next, which would suffice if $d s$ is chosen to be small enough. The optimum value of $d s$ clearly depends on the local curvature, which varies along the field line. However, the problem is approached in a different way in the program. It is assumed that the field line is dipolar between successive points, so that the field line traced is a succession of dipolar elements of arc length $d s$. The value of $\boldsymbol{B}$ at each point on the trace is calculated from the geomagnetic-field model, and the dipole axis corresponding to the next element is orientated to give a dipole field at that point parallel to the field of the model. This dipole axis will change slightly at each step.

Each location is stored in the program as a colatitude, longitude and height, so that the task is to calculate the coordinates of the next point on the trace. To accomplish this, the program first determines the location of the point with respect to the dipole axis by calculating the corresponding dipole co-latitude, $\vartheta$, from the relation

$2 \cot \vartheta=\tan I$

which is valid for a dipole field. The parameter $I$ is the inclination of the field at the point, which is easily calculated from $\boldsymbol{B}$. It depends only on the direction of the field and so is calculated from the geomagnetic-field model. With $\vartheta$ known, the position of the next point is found by calculating the change in $\vartheta$ that corresponds to $d s$. The increment $d \vartheta$ is calculated from the following equation 
$d \vartheta=\frac{r}{R_{\mathrm{E}}}\left[\frac{2 \sin \vartheta}{\sqrt{3 \cos ^{2} \vartheta+1}}\right] \frac{d s}{R_{\mathrm{E}}}$,

where $r$ is the geocentric distance (in $\mathrm{km}$ ) of the point (i.e. the magnitude of $\boldsymbol{r}$ ) and $R_{\mathrm{E}}$ is the mean radius of the Earth $(6371.2 \mathrm{~km})$, which is assumed to be spherical. A value of $d s$ (in $\mathrm{km}$ ) is required, but this remains fixed throughout the trace. The authors have minor reservations about the precise functional form of Eq. 8, in the sense that it may require the use of a value of $d s$ that is smaller than it actually needs to be [since there are theoretical grounds for believing that the right-hand side of Eq. 8 should be multiplied by the factor $\left.\left(R_{\mathrm{E}} / r\right)^{2}\right]$.

It is now necessary to specify the present position in the coordinates employed by the program, which are a modified form of the spherical polar coordinates $r, \theta$ and $\phi$. The main difference is the use of the height, $h$, which is simply related to $r$ by the equation

$h=r-R_{\mathrm{E}}$,

since the Earth is assumed to be spherical. The value of $r$ for the new position is easily found by using the equation for the field lines of a magnetic dipole (cf. Eq. 5 of Paper I)

$r=r_{1} \sin ^{2} \vartheta$.

The procedure is first to obtain the constant $r_{1}$ from $r$ and $\vartheta$ at one point and then calculate $r$ at the next point from $\vartheta+d \vartheta$ and $r_{1}$. The value of $h$ at the new position is found from Eq. 9. The other two coordinates, $\theta$ and $\phi$, are computed as increments, $d \theta$ and $d \phi$, from one point to the next. The relevant equations are

$d \theta=\cos D d \vartheta$

and

$d \phi=\frac{\sin D}{\sin \theta} d \vartheta$

where $D$ is the magnetic declination, which is again calculated from $\boldsymbol{B}$. As the components of this vector are not yet known at the new position, those of the previous point are used. These two increments are then used to calculate new values of $\theta$ and $\phi$, and hence the components of $\boldsymbol{B}$ are found at the new point. From these magnetic-field components, values of $I$ and $D$ are calculated for this new point and the cycle is repeated.

The procedure can be summarized schematically as follows:

(a) Start with coordinates $(h, \theta, \phi)$ at a given point;

(b) calculate the value of $r$ corresponding to $h$ using Eq. 9;

(c) obtain the components of $\boldsymbol{B}$, and hence $D$ and $I$, for this point;

(d) determine $\vartheta$ from Eq. 7 and then $d \vartheta$ from Eq. 8;

(e) obtain the constant $r_{1}$ in Eq. 10 from $r$ and $\vartheta$ at this point;

(f) use this constant again in Eq. 10 to calculate $r$ at $\vartheta+d \vartheta$

(g) compute the value of $h$ corresponding to the new $r$ from Eq. 9; (h) determine $d \theta$ and $d \phi$ from Eqs. 11 and 12, respectively;

(j) obtain new values of $\theta$ and $\phi$ from $d \theta$ and $d \phi$;

(k) calculate the components of $\boldsymbol{B}$ at the new point and repeat the cycle.

\subsection{Calculation of the magnetic-field components}

In the system of spherical polar coordinates defined in Sect. 2.1, the components of the magnetic field $\boldsymbol{B}$ used in the previous section are defined by Eqs. 1-3. The magnetic-field components can be obtained in other coordinate systems by means of suitable coordinate transformations. The standard version of the computer program calculates magnetic-field components using the spherical harmonic coefficients for the sixth generation of the International Geomagnetic Reference Field, here abbreviated to IGRF6 (Langel, 1991, 1992). Tables of spherical harmonic coefficients can be calculated for any epoch between 1945 and 1990, with linear extrapolation beyond (cf. Sec. 2.2 of Paper I). Two different sets of routines have been used to calculate $\boldsymbol{B}$ from such spherical harmonic coefficients. One set of routines is based on the approach adopted by Kluge (1970 a,b,c, 1972): the other is based on an algorithm for synthesizing the geomagnetic field developed by Malin and Barraclough (1981).

The standard version of the program employs the Kluge routines, makes use of all the IGRF6 coefficients for the required epoch and has the value of $d s$ fixed at 20 $\mathrm{km}$. The following modifications have been made for the present work on the uncertainties in magnetic-field-line tracing in the magnetosphere:

(i) The value of $d s$ is now read by the program and can therefore be varied from trace to trace, but is kept fixed during each individual trace. A value of 0.01 $\mathrm{km}$ has been used in the present calculations.

(ii) Separate versions of the computer program have been set up to use either the Kluge or the Malin and Barraclough routines.

(iii) The spherical harmonic expansion can be truncated so that $\boldsymbol{B}$ is calculated for each of the ten degrees of the IGRF6 model $\left(1 \leq n_{\max } \leq 10\right)$; the simplest of these corresponds to a dipole field $\left(n_{\max }=1\right)$.

In course of this work it was found that the Kluge routines fail in the dipole case; therefore, the Malin and Barraclough routines have been used exclusively in this investigation. Moreover, the actual spherical harmonic coefficients used in this paper are those of the NASA Goddard Space Flight Center Model designated GSFC 1990D (Langel et al., 1992), rather than those of IGRF6 (Langel, 1991, 1992). This change is necessitated by the fact that a knowledge of the standard errors in the coefficients is a prerequisite in the present study. The values of the spherical harmonic coefficients and their standard errors for GSFC 1990D are presented in Appendix B. These values have been used in all illustrative numerical calculations of the uncertainty in field-line tracing in the magnetosphere that are presented in this paper. 


\section{An algorithm that curtails the computing time}

As indicated in Sect. 2.2 and summarized succinctly in Appendix A, evaluating $\delta r_{\max }$ by considering all permutations of the end-points of the uncertainty intervals for the spherical harmonic coefficients is only practicable computationally in the case of the axisymmetric part $(m=0)$ of the internal geomagnetic field (i.e. $g_{n}^{0} \pm \delta g_{n}^{0}$ for $\left.1 \leq n \leq 10\right)$. For the complete internal geomagnetic field, the all-permutations approach is not really feasible beyond $n_{\max }=3$ (i.e. $g_{n}^{m} \pm \delta g_{n}^{m}$ and $h_{n}^{m} \pm \delta h_{n}^{m}$ for $0 \leq m \leq n$ and $\left.1 \leq n \leq 3\right)$. An algorithm that relaxes this restriction is formulated in Sect. 4.1. Although no mathematical proof of the validity of this algorithm has yet been found, it is shown in Sect. 4.2 that the algorithmic and permutational approaches yield identical results in those cases for which such comparisons are computationally feasible. The comparative cases considered are (i) the general axisymmetric part of the internal geomagnetic field $\left(m=0, n_{\max }=10\right)$ and (ii) the complete (non-axisymmetric) internal geomagnetic field for the restrictive case in which fourth- and higherdegree multipoles are neglected $\left(m \neq 0, n_{\max }=3\right)$. Illustrative numerical estimates of the maximum uncertainty in field-line tracing from the surface of the Earth to the magnetic equator are presented in Sec. 4.3.

\subsection{Formulation of the algorithm}

The formulation of the algorithm may be specified as a sequence of operations as follows:

(i) Express the spherical harmonic coefficients as an ordered set of $N$ terms in the conventional way; namely $\left\{g_{1}^{0}, g_{1}^{1}, h_{1}^{1}, g_{2}^{0}, g_{2}^{1}, h_{2}^{1}, g_{2}^{2}, h_{2}^{2}, g_{3}^{0}, \cdots, g_{n_{\max }}^{n_{\max }}, h_{n_{\max }}^{n_{\max }}\right\}$, where $N$ $=n_{\max }\left(n_{\max }+2\right)$

(ii) Let $\mathscr{G}_{i}^{\mathscr{d}}$ be a $(1 \times N)$ array of ordered spherical harmonic coefficients that represents the $i^{\text {th }}$ geomagnetic-field model from the set of $(N+1)$ geomagneticfield models $\left\{\mathscr{G}_{i}^{\mathscr{A}}\right\}(i=1,2, \cdots, N+1)$, defined by the following operations. Let $\delta r_{i}^{d}$ denote the magnitude of the corresponding vector uncertainty $\boldsymbol{\delta}_{i}^{\mathscr{A}}$ (as defined in Sect. 2.2) in the location of a "reference point" on a magnetic field line, so that the magnitude of this uncertainty is measured relative to $\boldsymbol{\delta} \boldsymbol{r}=0$ for the central geomagnetic-field model $\mathscr{G}_{0}^{\mathscr{A}}\left(=\mathscr{G}_{0}\right.$, defined by the set of central values $g_{n}^{m}$ and $h_{n}^{m}$, as in Sect. 2.4 of Paper I).

(iii) Choose the elements of $\mathscr{G}_{1}^{\mathscr{A}}$ to be $\left(g_{n}^{m}+\delta g_{n}^{m}\right.$, $\left.h_{n}^{m}+\delta h_{n}^{m}\right)$ for $0 \leq m \leq n, 1 \leq n \leq n_{\max }, \delta g_{n}^{m}>0$ and $\delta h_{n}^{m}>0$, and calculate $\delta r_{1}^{d}$ (i.e, choose the "upper" end-points for all spherical harmonic coefficients).

(iv) Define $\mathscr{G}_{2}^{\mathscr{A}}$ such that the sign of the uncertainty $\left(\delta g_{1}^{0}\right)$ in just the first spherical harmonic coefficient $\left(g_{1}^{0}\right)$ is changed from a plus $(+)$ to a minus $(-)$ and the signs of all other coefficients remain unchanged (i.e. + ). Calculate $\delta r_{2}^{d}$ for $\mathscr{G}_{2}^{\mathscr{d}}$.

(v) Compare $\delta r_{2}^{d}$ with $\delta r_{1}^{d}$ and select the geomagnetic-field model with the larger uncertainty. Suppose, for example, that $\delta r_{1}^{d}>\delta r_{2}^{d}$ so that $\mathscr{G}_{1}^{\mathscr{d}}$ is selected (i.e. $g_{1}^{0}+\delta g_{1}^{0}$ is accepted and $g_{1}^{0}-\delta g_{1}^{0}$ is rejected). (vi) Define $\mathscr{G}_{3}^{\mathscr{A}}$ such that it only differs from $\mathscr{G}_{1}^{\mathscr{A}}$ by having the sign of the uncertainty $\left(\delta g_{1}^{1}\right)$ in just the second spherical harmonic coefficient $\left(g_{1}^{1}\right)$ changed from a plus $(+)$ to a minus $(-)$. Calculate $\delta r_{3}^{\mathscr{A}}$ for $\mathscr{G}_{3}^{\mathscr{A}}$.

(vii) Compare $\delta r_{3}^{d}$ with $\delta r_{1}^{d}$ and suppose, for example, that $\delta r_{3}^{\mathscr{d}}>\delta r_{1}^{\mathscr{A}}$ so that $\mathscr{G}_{3}^{\mathscr{A}}$ is selected (i.e. $g_{1}^{1}-\delta g_{1}^{1}$ is accepted and $g_{1}^{1}+\delta g_{1}^{1}$ is rejected).

(viii) Continue this procedure, with the sense of the comparisons always proceeding from left to right through the ordered set of spherical harmonic coefficients specified in (i).

(ix) At the end of the $(N+1)$ comparisons, accept the model $\mathscr{G}_{N+1}^{\mathscr{A}}\left(=\mathscr{G}_{\max }^{\mathscr{A}}\right)$ that gives the largest uncertainty $\delta r_{N+1}^{\infty}$. It is assumed that this limiting geomagnetic-field model, $\mathscr{G}_{N+1}^{\mathscr{d}}$, is identical to the limiting geomagnetic-field model, $\mathscr{G}_{\max }$, that would be obtained if it were possible to consider all permutations of the "end-points" of the spherical harmonic coefficients (i.e. $g_{n}^{m} \pm \delta g_{n}^{m}$ and $h_{n}^{m} \pm \delta h_{n}^{m}$ for $0 \leq m \leq n$ and $1 \leq n \leq n_{\max }$ ). Accordingly, it is assumed that $\delta r_{N+1}^{d}\left(=\delta r_{\max }^{\mathscr{A}}\right)$ is identical to $\delta r_{\max }$.

The sequence of operations involved in the application of this algorithm is illustrated schematically as follows in the general case $N=n_{\max }\left(n_{\max }+2\right)$ :

$$
\begin{aligned}
& \mathscr{G}_{1}^{\mathscr{A}}=\left[g_{1}^{0}+\delta g_{1}^{0}, g_{1}^{1}+\delta g_{1}^{1}, h_{1}^{1}+\delta h_{1}^{1}, g_{2}^{0}\right. \\
& +\delta g_{2}^{0}, \cdots, g_{n_{\max }}^{n_{\max }} \\
& \left.+\delta g_{n_{\max }}^{n_{\max }}, h_{n_{\max }}^{n_{\max }}+\delta h_{n_{\max }}^{n_{\max }}\right] \rightarrow \delta r_{1}^{\mathscr{d}}, \\
& \mathscr{G}_{2}^{\mathscr{A}}=\left[g_{1}^{0}-\delta g_{1}^{0}, g_{1}^{1}+\delta g_{1}^{1}, h_{1}^{1}+\delta h_{1}^{1}, g_{2}^{0}\right. \\
& +\delta g_{2}^{0}, \cdots, g_{n_{\max }}^{n_{\max }} \\
& \left.+\delta g_{n_{\max }}^{n_{\max }}, h_{n_{\max }}^{n_{\max }}+\delta h_{n_{\max }}^{n_{\max }}\right] \rightarrow \delta r_{2}^{\mathscr{A}}, \\
& \mathscr{G}_{3}^{\mathscr{A}}=\left[g_{1}^{0} \oplus \delta g_{1}^{0}, g_{1}^{1}-\delta g_{1}^{1}, h_{1}^{1}+\delta h_{1}^{1}, g_{2}^{0}\right. \\
& +\delta g_{2}^{0}, \cdots, g_{n_{\max }}^{n_{\max }} \\
& \left.+\delta g_{n_{\max }}^{n_{\max }}, h_{n_{\max }}^{n_{\max }}+\delta h_{n_{\max }}^{n_{\max }}\right] \rightarrow \delta r_{3}^{\mathscr{d}}, \\
& \mathscr{G}_{4}^{\mathscr{A}}=\left[g_{1}^{0} \oplus \delta g_{1}^{0}, g_{1}^{1} \ominus \delta g_{1}^{1}, h_{1}^{1}-\delta h_{1}^{1}, g_{2}^{0}\right. \\
& +\delta g_{2}^{0}, \cdots, g_{n_{\max }}^{n_{\max }} \\
& \left.+\delta g_{n_{\max }}^{n_{\max }}, h_{n_{\max }}^{n_{\max }}+\delta h_{n_{\max }}^{n_{\max }}\right] \rightarrow \delta r_{4}^{\mathscr{A}}, \\
& \mathscr{G}_{N+1}^{\mathscr{A}}=\left[g_{1}^{0} \oplus \delta g_{1}^{0}, g_{1}^{1} \ominus \delta g_{1}^{1}, h_{1}^{1} \ominus \delta h_{1}^{1}, g_{2}^{0}\right. \\
& \oplus \delta g_{2}^{0}, \cdots, g_{n_{\max }}^{n_{\max }} \\
& \left.\ominus \delta g_{n_{\max }}^{n_{\max }}, h_{n_{\max }}^{n_{\max }} \oplus \delta h_{n_{\max }}^{n_{\max }}\right] \rightarrow \delta r_{N+1}^{d} .
\end{aligned}
$$

The symbols $\oplus$ and $\ominus$ are used in this sequence of operations to denote illustratively positive and negative signs that are already determined (fixed) by a previous comparison between $\delta r_{i}^{\mathscr{A}}$ and $\delta r_{j}^{\mathscr{A}}(l \leq i<j, j=$ $2,3, \cdots, N+1)$. Therefore, the algorithm involves $N+2$ field-line tracings: one field-line tracing is associated with the central geomagnetic-field model $\mathscr{G}_{0}$ and then two tracings are required to determine the sign $( \pm)$ of the uncertainty $\delta g_{1}^{0}$ in the first spherical harmonic 
coefficient $\left(g_{1}^{0}\right)$. A further $(N-1)$ field-line tracings are required to determine the signs of the uncertainties in the remaining $(N-1)$ spherical harmonic coefficients. Hence the algorithm requires $N+2$ field-line tracings, whereas considering all permutations of the "end-points" of the spherical harmonic coefficients (i.e. $g_{n}^{m} \pm \delta g_{n}^{m}$ and $h_{n}^{m} \pm \delta h_{n}^{m}$ for $0 \leq m \leq n$ and $\left.1 \leq n \leq n_{\max }\right)$ requires $2^{N}+1$ field-line tracings (cf. Sect. 2.2).

\subsection{Comparison between the algorithmic and permutational procedures}

In the case of the complete internal geomagnetic field $\left(m \neq 0, n_{\max }=10\right), N=120$ and 122 field-line tracings are required in the algorithmic procedure, compared with $2^{120}+1$ field-line tracings in the permutational procedure. This reduction in the number of field-line tracings represents a stupendous curtailment in computing time from the order of $10^{28}$ years on a Cray Y - MP computer for the permutational procedure, as indicated in Appendix A, to about 5 min on a DEC 3000 AXP (Alpha)-Model 400 computer for the algorithmic procedure. The agreement between the algorithmic and permutational procedures can be checked rigorously in two special cases: (i) the axisymmetric part $(m=0)$ of the internal geomagnetic field in the general case $N=n_{\max }=10$ and (ii) the complete $(m \neq 0)$ internal geomagnetic field in the restrictive case $n_{\max }=3$, $N=15$. In case (i) the algorithmic procedure requires 12 tracings, whereas the permutational procedure requires 1025 tracings: in case (ii) the corresponding numbers are 17 and 32769 . The checks are performed by calculating $\delta r_{\max }$ according to the permutational procedure described in Sect. 2.2 and calculating $\delta r_{\max }^{d}$ according to the algorithmic procedure described in Sect. 4.1. These computations indicate that $\delta r_{\max }^{\mathscr{A}}=\delta r_{\max }$ to an accuracy of six decimal places, which confirms the validity of the algorithmic procedure in both cases for which a strict comparison between the two numerical procedures is practicable in terms of computing resources. On the basis of these two successful comparisons, it is assumed that the algorithmic procedure can be used with confidence in those cases for which the permutational procedure involves prohibitively long computing times.

The success of the algorithm described in Sect. 4.1 depends on the fact that the magnitudes of the spherical harmonic coefficients in the ordered set $\left\{g_{1}^{0}, g_{1}^{1}, h_{1}^{1}, g_{2}^{0}, g_{2}^{1}, h_{2}^{1}, g_{2}^{2}, h_{2}^{2}, g_{3}^{0}, \cdots, g_{n_{\max }}^{n_{\max }}, h_{n_{\max }}^{n_{\max }}\right\}$ decrease more or less steadily as the degree $(n)$ increases. It has been found that the algorithm fails if the spherical harmonic coefficients are considered in reverse order, so that the signs of the uncertainties in the smallest spherical harmonic coefficients $(n=10)$ are determined first. Although it would undoubtedly be of some interest to establish how far the ordered set of coefficients can be permuted before the algorithm fails (perhaps even without moving the dipole out of the first position), this question is not considered in the present paper. Such an extension is not strictly necessary, however, because it has already been shown that the algorithmic procedure defined in Sect. 4.1 actually gives identical results to the permutational procedure in all cases in which this comparison can be checked with reasonable computing resources.

\subsection{Numerical estimates of the maximum uncertainty in field-line tracing}

Table 1 presents numerical estimates of the maximum uncertainty $\delta r_{\max }\left(=\delta r_{\max }^{d}\right)$ in the geocentric distance at which a magnetic field line crosses the geomagnetic equator $(\theta=\pi / 2)$ for the axisymmetric part $(m=0)$ of the geomagnetic field of internal origin $\left(N=n_{\max }=10\right)$. As in Paper I, results are presented for nominal (dipolar) equatorial crossing distances of $r_{1}=2 R_{\mathrm{E}}$ and $r_{1}=6 R_{\mathrm{E}}$. The values presented in Table 1 repeat those given in Table 4 of Paper I for the tracing program, although it should be stressed here that the algorithmic and permutational procedures give identical results for $\delta r_{\max }$ (to six decimal places). Moreover, the estimates of $\delta r_{\max }$ obtained using the tracing program are in excellent agreement with those obtained independently in Paper I (cf. Table 4) from the solution of the polynomial equation $\left(n_{\max }=10\right)$ for the magnetic field lines of the axisymmetric part $(m=0)$ of the internal geomagnetic field. The field-line tracing program is therefore assumed to be equally accurate in determining the magnetic field lines of the complete (non-axisymmetric) geomagnetic field of internal origin.

Having established the accuracy of the magneticfield-line tracing program, it is possible to present numerical estimates for $\delta r_{\max }$ in the case of the complete internal geomagnetic field. However, since the complete internal geomagnetic field is non-axisymmetric, the uncertainties in field-line tracing in the magnetosphere now depend on both the co-latitude $(\theta)$ and longitude $(\phi)$ of the starting point. In this paper, illustrative numerical results are presented for the two special geographic longitudes defined by the centred geomagnetic dipole axis (i.e. defined by $g_{1}^{0}, g_{1}^{1}$ and $h_{1}^{1}$ ). These two special geographic longitudes, which are referred to as Longitude 1 and Longitude 2, pass through the respective directions, $\left(\theta_{1}, \phi_{1}\right)$ and $\left(\theta_{2}, \phi_{2}\right)$, defined as follows:

$\begin{aligned} \text { Longitude 1: } & \theta_{1}=\arccos \llbracket\left|g_{1}^{0}\right| /\left[\left(g_{1}^{0}\right)^{2}+\left(g_{1}^{1}\right)^{2}+\left(h_{1}^{1}\right)^{2} \rrbracket^{1 / 2} \rrbracket\right. \\ \phi_{1} & =\arctan \left(h_{1}^{1} / g_{1}^{1}\right)\end{aligned}$

and

Table 1. Numerical estimates for $\delta r_{\max }$ at the geomagnetic equator in the case of the axisymmetric part of the internal geomagnetic field $\left(n_{\max }=10, N=10\right)$, obtained using the field-line tracing program $\left({ }^{*} \mathscr{A}=\right.$ algorithmic, $\mathscr{P}=$ permutational $)$

\begin{tabular}{llll}
\hline$n_{\text {max }}$ & $\begin{array}{l}\delta r_{\text {max }}\left(r_{1}=2 R_{\mathrm{E}}\right) \\
(\mathrm{km})\end{array}$ & $\begin{array}{l}\delta r_{\text {max }}\left(r_{1}=6 R_{\mathrm{E}}\right) \\
(\mathrm{km})\end{array}$ & $\begin{array}{l}\text { Procedure } \\
\left({ }^{*} \mathscr{A}, \mathscr{P}\right)\end{array}$ \\
\hline 10 & 9.30 & 65.48 & $\mathscr{A}$ or $\mathscr{P}$ \\
\hline
\end{tabular}


Longitude 2: $\quad \theta_{2}=\pi-\arccos \llbracket\left|g_{1}^{0}\right| / \llbracket\left(g_{1}^{0}\right)^{2}$

$$
\begin{gathered}
\left.+\left(g_{1}^{1}\right)^{2}+\left(h_{1}^{1}\right)^{2}\right]^{1 / 2} \rrbracket \\
\phi_{2}=\pi+\arctan \left(h_{1}^{1} / g_{1}^{1}\right) .
\end{gathered}
$$

Tables 2 and 3 present numerical estimates, for Longitudes 1 and 2 respectively, of the maximum uncertainty $\delta r_{\max }\left(=\delta r_{\max }^{\mathscr{d}}\right)$ in the geocentric distance at which a magnetic field line crosses the geomagnetic equator $\left(\theta-\theta_{1}=\pi / 2\right.$ or $\left.\theta_{2}-\theta=\pi / 2\right)$ for the complete geomagnetic field of internal origin $\left(n_{\max }=\right.$ $10, N=120)$. Once again, results are presented for nominal (dipolar) equatorial crossing distances of $r_{1}=2$ and $6 R_{\mathrm{E}}$. All these results have been derived using the algorithmic $(\mathscr{A})$ procedure and those for which $1 \leq n_{\max } \leq 3$ have been checked using the permutational $(\mathscr{P})$ procedure. Small differences in the numerical values of $\delta r_{\max }(<2 \%)$ occur if geocentric $\left(r=R_{\mathrm{E}}\right)$ rather than true geodetic starting latitudes are derived using the relations $r_{1}=2 R_{\mathrm{E}}$ and $r_{1}=6 R_{\mathrm{E}}$. Since the magnetic field of a dipole $(n=1)$ is axisymmetric, the values of $\delta r_{\max }$ for $n_{\max }=1$ should be the same at the same geocentric latitude for Longitudes 1 and 2 (for both $r_{1}=2$ and $\left.6 R_{\mathrm{E}}\right)$. The very small differences $(<0.8 \%)$ between the corresponding numbers in Tables 2 and 3 result from the conversion from geocentric to geodetic starting coordinates. Tables 2 and 3 indicate that $\delta r_{\max }$ increases monotonically as $n_{\max }$ increases from 1 to 10 , apart from the small negative increment from $n_{\max }=4$ to $n_{\max }=5$ for Longitude 1 and $r_{1}=2 R_{\mathrm{E}}$. The values of $\delta r_{\max }$ for $n_{\max }=10$ are substantially larger for the complete internal geomagnetic field (Tables 2 and 3) than for just the axisymmetric part (Table 1), which is a direct consequence of the fact that there are 120 standard errors for the complete field, compared with only 10 for the axisymmetric part.

The numerical estimates for $\delta r_{\max }$ presented in Tables 1-3 are calculated using the internal geomagnetic-field model designated GSFC 1990D: the spherical harmonic coefficients $\left(g_{n}^{m}, h_{n}^{m}\right)$ and their corresponding standard errors $\left(\delta g_{n}^{m}, \delta h_{n}^{m}\right)$ are given in Appendix B. As noted in Sect. 5 of Paper I, if each $\delta g_{n}^{m}$ and $\delta h_{n}^{m}$ is taken to be the same multiple (say $\lambda$ ) of the standard error, then the

Table 2. Numerical estimates for $\delta r_{\max }$ at the geomagnetic equator in the case of the complete internal geomagnetic field $\left(n_{\max } 10\right.$, $N=120$ ), obtained using the field-line tracing program for Longitude $1(* \mathscr{A}=$ algorithmic, $\mathscr{P}=$ permutational $)$

\begin{tabular}{rlcl}
\hline$n_{\text {max }}$ & $\begin{array}{l}\delta r_{\max }\left(r_{1}=2 R_{\mathrm{E}}\right) \\
(\mathrm{km})\end{array}$ & $\begin{array}{l}\delta r_{\max }\left(r_{1}=6 R_{\mathrm{E}}\right) \\
(\mathrm{km})\end{array}$ & $\begin{array}{l}\text { Procedure } \\
\left({ }^{*} \mathscr{A}, \mathscr{P}\right)\end{array}$ \\
\hline 1 & 14.08 & 94.11 & $\mathscr{A}$ or $\mathscr{P}$ \\
2 & 30.26 & 134.29 & $\mathscr{A}$ or $\mathscr{P}$ \\
3 & 45.67 & 215.73 & $\mathscr{A}$ or $\mathscr{P}$ \\
4 & 57.78 & 270.36 & $\mathscr{A}$ \\
5 & 56.85 & 297.98 & $\mathscr{A}$ \\
6 & 64.03 & 322.33 & $\mathscr{A}$ \\
7 & 76.35 & 339.13 & $\mathscr{A}$ \\
8 & 79.95 & 354.71 & $\mathscr{A}$ \\
9 & 83.39 & 368.75 & $\mathscr{A}$ \\
10 & 86.25 & 380.65 & \\
\hline
\end{tabular}

Table 3. Numerical estimates for $\delta r_{\max }$ at the geomagnetic equator in the case of the complete internal geomagnetic field $\left(n_{\max }=10\right.$, $N=120$ ), obtained using the field-line tracing program for Longitude $2(* \mathscr{A}=$ algorithmic, $\mathscr{P}=$ permutational $)$

\begin{tabular}{rlcl}
\hline$n_{\max }$ & $\begin{array}{l}\delta r_{\max }\left(r_{1}=2 R_{\mathrm{E}}\right) \\
(\mathrm{km})\end{array}$ & $\begin{array}{l}\delta r_{\max }\left(r_{1}=6 R_{\mathrm{E}}\right) \\
(\mathrm{km})\end{array}$ & $\begin{array}{l}\text { Procedure } \\
\left({ }^{*} \mathscr{A}, \mathscr{P}\right)\end{array}$ \\
\hline 1 & 14.01 & 93.43 & $\mathscr{A}$ or $\mathscr{P}$ \\
2 & 31.65 & 201.70 & $\mathscr{A}$ or $\mathscr{P}$ \\
3 & 48.71 & 313.65 & $\mathscr{A}$ or $\mathscr{P}$ \\
4 & 63.49 & 437.08 & $\mathscr{A}$ \\
5 & 70.59 & 483.76 & $\mathscr{A}$ \\
6 & 76.85 & 528.76 & $\mathscr{A}$ \\
7 & 80.65 & 553.34 & $\mathscr{A}$ \\
8 & 84.33 & 573.06 & $\mathscr{A}$ \\
9 & 87.69 & 589.35 & $\mathscr{A}$ \\
10 & 90.60 & 603.07 & $\mathscr{A}$ \\
\hline
\end{tabular}

associated numerical uncertainties can be obtained approximately by multiplying the tabulated values of $\delta r_{\max }$ by the factor $\lambda$.

\section{Interval mapping for the complete internal geomagnetic field}

As in Paper I, it is assumed implicitly in Sect. 2.2 that in the case of the complete internal geomagnetic field $(m \neq 0) \delta r_{\max }$ can be determined by considering every possible permutation of the "lower" and "upper" endpoints, $g_{n}^{m}-\delta g_{n}^{m}, h_{n}^{m}-\delta h_{n}^{m}, g_{n}^{m}+\delta g_{n}^{m}$ and $h_{n}^{m}+\delta h_{n}^{m}$, of the intervals of real numbers $\left[g_{n}^{m}-\delta g_{n}^{m}, g_{n}^{m}+\delta g_{n}^{m}\right]$ and $\left[h_{n}^{m}-\delta h_{n}^{m}, h_{n}^{m}+\delta h_{n}^{m}\right]$ for $0 \leq m \leq n$ and $1 \leq n \leq n_{\max }$. Stated alternatively, it is assumed that the maximum value of $\delta r\left(\delta r_{\max }\right)$ in the set of values $\{r+\delta r\}$ corresponds to a particular permutation of the end-points $\left(g_{n}^{m} \pm \delta g_{n}^{m}\right),\left(h_{n}^{m} \pm \delta h_{n}^{m}\right) \quad$ in the coefficient intervals $\left[g_{n}^{m}+\Delta g_{n}^{m}\right],\left[h_{n}^{m}+\Delta h_{n}^{m}\right]$, where $\delta r \in \mathbb{R}_{0}^{+}, \quad-\delta g_{n}^{m} \leq \Delta g_{n}^{m}$ $\leq+\delta g_{n}^{m}$ and $-\delta h_{n}^{m} \leq \Delta h_{n}^{m} \leq+\delta h_{n}^{m}$. This implicit assumption effectively reduces a continuous interval mapping problem to a discrete one. Since it is intuitively clear that the complete spherical harmonic expansion of the internal geomagnetic field is a well-behaved function of the spherical harmonic coefficients, this implicit assumption appears reasonable physically. However, even with this assumption, the computing time required to calculate $\delta r_{\max }$ in the case of the complete internal geomagnetic field is prohibitively long for $n_{\max } \geq 4$ (cf. Sect. 2.2 and Appendix A). Therefore, an algorithm that essentially eliminates the need to consider every possible permutation of the "lower" and "upper" end-points is introduced in Sect. 4.1.

\subsection{Conjecture 2: interval mapping in the non-axisymmetric case $(\mathrm{m} \neq 0)$}

Consider the following conjecture for the complete internal geomagnetic field $(m \neq 0)$, which is designated Conjecture 2. (Conjecture 1 is presented in Paper I.) Conjecture 2: For the contemporary geomagnetic field, there do not exist points $g_{n}^{m}+\Delta g_{n}^{m}$ and $h_{n}^{m}+\Delta h_{n}^{m}$, where $-\delta g_{n}^{m} \leq \Delta g_{n}^{m} \leq+\delta g_{n}^{m}\left(\delta g_{n}^{m} \geq 0\right)$ and $-\delta h_{n}^{m} \leq$ 


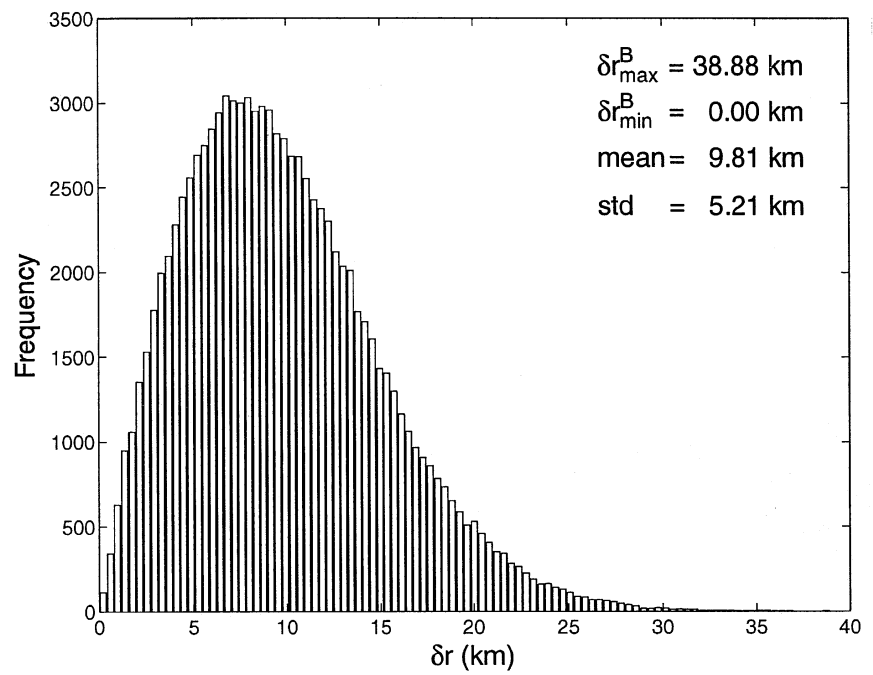

a

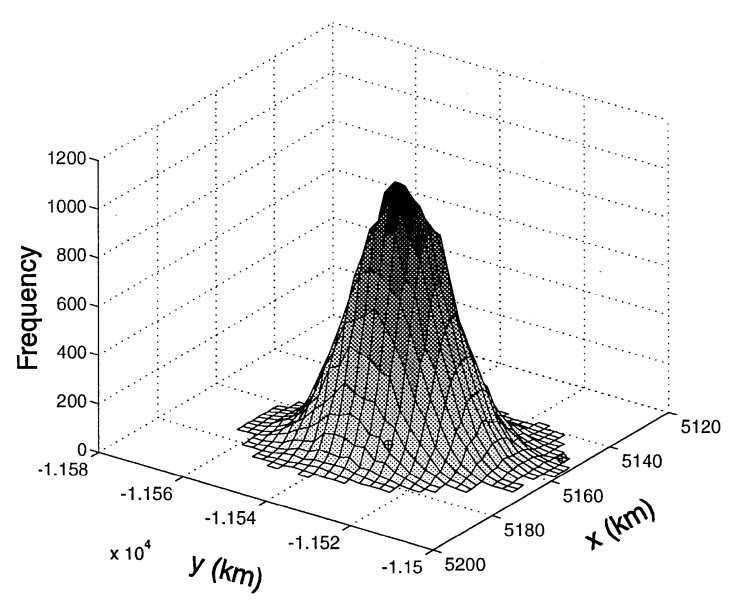

c

Isometric Projection

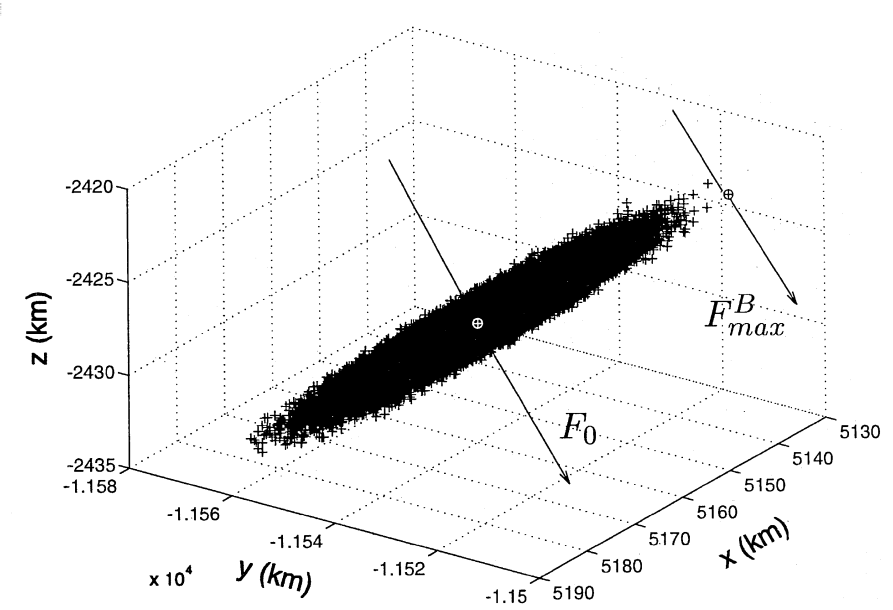

b

Scatter Plot

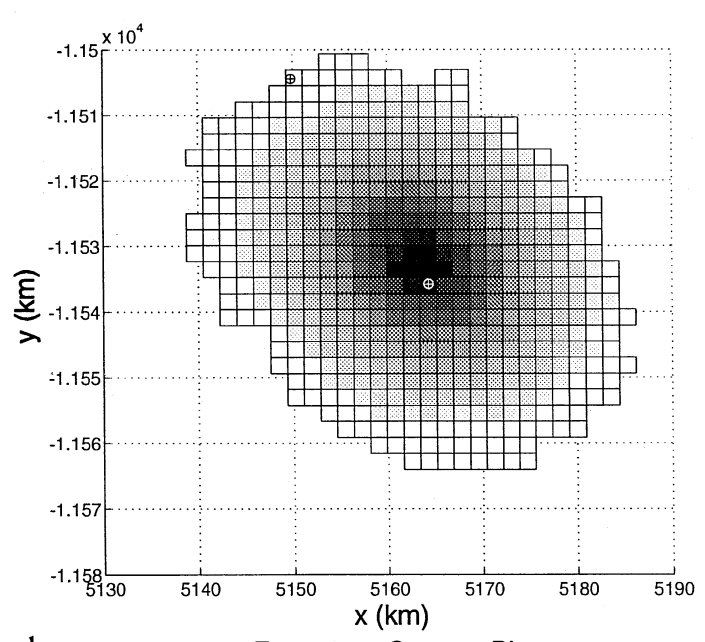

Frequency

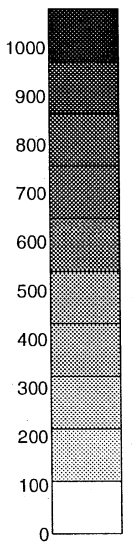

Fig. 1a-d. Plots of the uncertainties $(\delta r)$ in the geocentric distance at which a magnetic field line crosses the geomagnetic equator for Longitude 1 and $r_{1}=2 R_{\mathrm{E}}$ in the case of the complete internal geomagnetic field $\left(n_{\max }=10, N=120\right)$. a histogram of the uncertainties calculated by Method B, which involves $10^{5}$ field-line tracings associated with $10^{5}$ selections of 120 (random) errors $\Delta g_{n}^{m}$ and $\Delta h_{n}^{m}$ in $g_{n}^{m}$ and $h_{n}^{m}(0 \leq m \leq n, 1 \leq n \leq 10)$, located randomly in the 120

$\Delta h_{n}^{m} \leq+\delta h_{n}^{m}\left(\delta h_{n}^{m} \geq 0\right)$, in the respective intervals $\left[g_{n}^{m}-\delta g_{n}^{m}, g_{n}^{m}+\delta g_{n}^{m}\right]$ and $\left[h_{n}^{m}-\delta h_{n}^{m}, h_{n}^{m}+\delta h_{n}^{m}\right]$ for which $\mathscr{J}\left(g_{n}^{m}+\Delta g_{n}^{m}, h_{n}^{m}+\Delta h_{n}^{m}\right)=r+\Delta r$ such that $\Delta r>\delta r_{\max }$ $(\delta r, \Delta r \geq 0 ; c . f$. Sect. 2.2). The symbol $\mathscr{J}$ is used to denote the "transformation" from coefficient intervals to uncertainty interval using the tracing program discussed in Sect. 3 to find $\Delta r$ for given $\Delta g_{n}^{m}$ and $\Delta h_{n}^{m}\left(0 \leq m \leq n, 1 \leq n \leq n_{\max }\right)$. Note that with this definition $\mathscr{J}\left(g_{n}^{m}, h_{n}^{m}\right)=\bar{r}$ and $\mathscr{J}\left(g_{n}^{m} \pm \delta g_{n}^{m}, h_{n}^{m} \pm \delta h_{n}^{m}\right)$ $=r+\delta r_{\max }$.

As in Paper I, the credibility of Conjecture 2 is established by calculating $\delta r_{\max }$ by two different methods and comparing the results as follows. In Method A the algorithm formulated in Sec. 4.1 is used to determine $\delta r_{\max }^{A}\left(=\delta r_{\max }^{\mathscr{A}}\right)$ for the GSFC 1990D model (c.f. Appendix $B$ ). In Method $B$ the field-line tracing program

corresponding error intervals $\left[-\delta g_{n}^{m},+\delta g_{n}^{m}\right]$ and $\left[-\delta h_{n}^{m},+\delta h_{n}^{m}\right]$; b scatter plot of the points where the $10^{5}$ field-line tracings cross the geomagnetic equator (cf. Sect. 4.2); c isometric projection (in the geographic equator) of the $10^{5}$ geomagnetic equatorial crossing points; d frequency contour plot (in the geographic equator) of the $10^{5}$ geomagnetic equatorial crossing points

described in Sect. 3 is used to perform $10^{5}$ tracings, in each of which the 120 spherical harmonic coefficients $g_{n}^{m}$ and $h_{n}^{m}(0 \leq m \leq n, 1 \leq n \leq 10)$ in the GSFC 1990D model are assumed to have random errors $\Delta g_{n}^{m}$ and $\Delta h_{n}^{m}$ in the respective error intervals $\left[-\delta g_{n}^{m},+\delta g_{n}^{m}\right]$ and $\left[-\delta h_{n}^{m},+\delta h_{n}^{m}\right]$. Using an appropriate MATLAB routine (MATLAB Reference Guide, 1992), the resulting $10^{5}$ values of $\delta r^{B}$ are arranged as a monotonic increasing sequence $\left\{\delta r_{\text {min }}^{B}, \ldots, \delta r_{\text {max }}^{B}\right\}$, where $\delta r_{\text {min }}^{B}$ and $\delta r_{\max }^{B}$ denote the smallest and largest values of $\delta r^{B}$ calculated by Method B. As noted in Sect. 2.2, in the case of the complete internal geomagnetic field $(m \neq 0) \delta r$ should really be regarded as a vector $\boldsymbol{\delta} \boldsymbol{r}$ that has both a magnitude $\delta r(|\delta r|)$ and an azimuthal angle with respect to the central vector $\boldsymbol{r}$. Hence $\delta r_{\text {min }}^{B}=0$, which is otherwise evident from the figures discussed in 


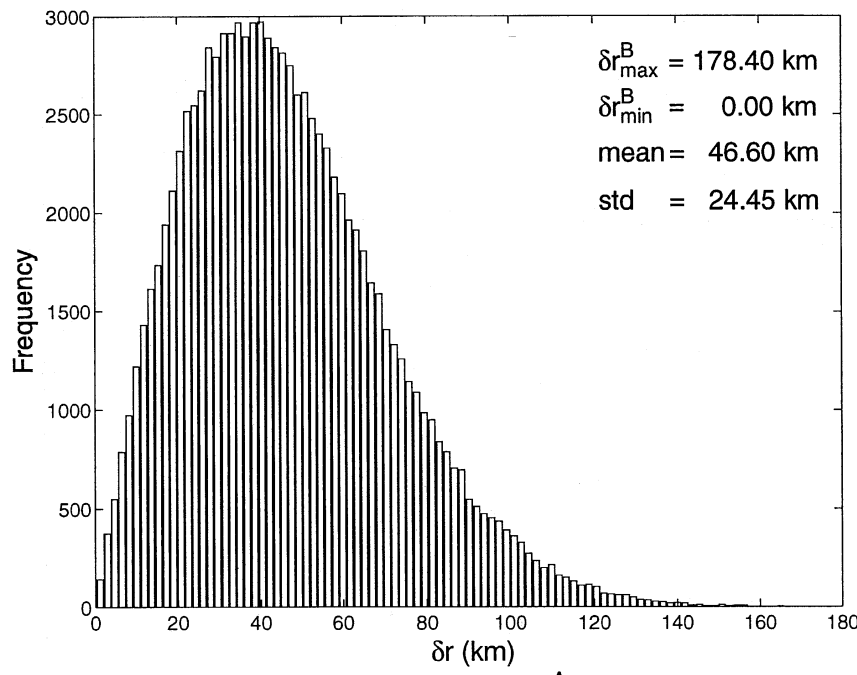

a

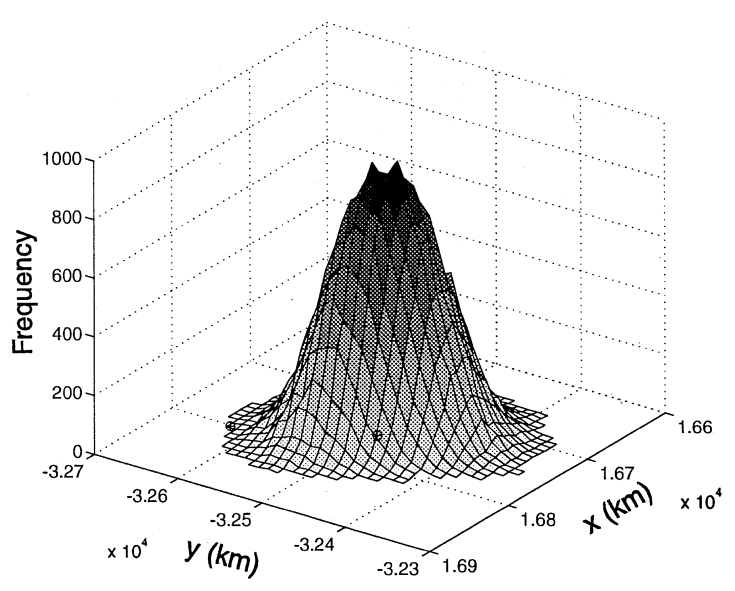

C

Isometric Projection

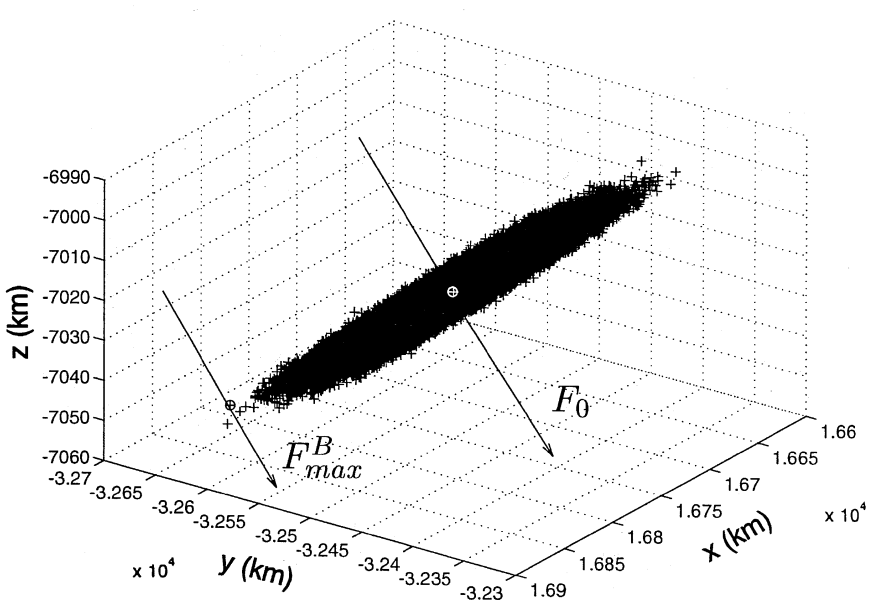

b

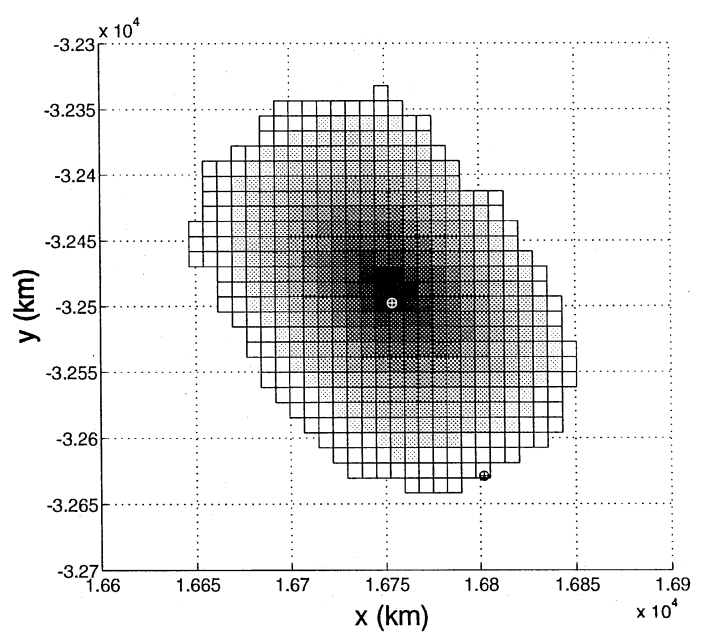

Frequency Contour Plot

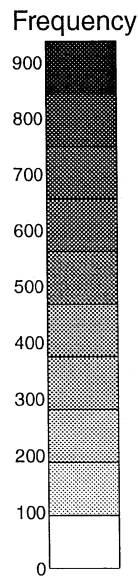

Fig. 2a-d. Same as Fig 1, but for Longitude 1 and $r_{1}=6 R_{\mathrm{E}}$

the following section. Lastly, the values of $\delta r_{\max }^{A}$ and $\delta r_{\max }^{B}$ are compared and Conjecture 2 is accepted (at least as a working hypothesis) if $\delta r_{\max }^{A}>\delta r_{\max }^{B}$. This condition has been satisfied in every case examined in this study. Therefore, a set of 120 internal points lying in the general coefficient intervals $\left[g_{n}^{m}-\delta g_{n}^{m}, g_{n}^{m}+\delta g_{n}^{m}\right]$ and $\left[h_{n}^{m}-\delta h_{n}^{m}, h_{n}^{m}+\delta h_{n}^{m}\right]$ for $0 \leq m \leq n, 1 \leq n \leq 10$ apparently always maps to a value of $\bar{\delta} r$ lying in the uncertainty interval $\left[0, \delta r_{\max }\right]$.

\subsection{Graphical presentation of results for the complete internal geomagnetic field}

The theoretical methods described in Sect. 5.1 are illustrated in Figs. 1-4, which correspond, respectively, to the four cases: (i) Longitude $1, r_{1}=2 R_{\mathrm{E}}$; (ii) Longitude $1, r_{1}=6 R_{\mathrm{E}}$; (iii) Longitude $2, r_{1}=2 R_{\mathrm{E}}$; and (iv) Longitude $2, r_{1}=6 R_{\mathrm{E}}$. Four panels are included in each of the four figures as follows: (a) a histogram of the monotonic increasing sequence $\left\{\delta r^{B}\right\}$ determined by Method B, described in Sect. 5.1; (b) a

scatter plot of the points where the $10^{5}$ field-line tracings discussed in Sect. 5.1 cross the geomagnetic equator; (c) an isometric projection in the geographic equator of the geomagnetic equatorial crossing points; and (d) a frequency contour plot in the geographic equator of these same points. The term "frequency" signifies absolute frequency in the statistical sense.

The histograms in Figs. 1a-4a are analogous to those presented in Figs. 1 and 2 of Paper I, although separate results are presented here for Longitudes 1 and 2. The actual numerical values of $\delta r_{\max }^{B}$ and $\delta r_{\min }^{B}(=0.00)$, as well as the mean and standard deviation (std), are included in the appropriate panel. The values of $\delta r_{\max }^{A}$ derived by Method A (the algorithmic procedure) are given in the captions to the histograms: these values are also given in Tables 2 and $3\left(n_{\max }=10\right)$. Comparisons between the actual numerical values confirm that $\delta r_{\max }^{A}>\delta r_{\max }^{B}$ in every case considered (including those presented in Sect. 6). Since $\delta r_{\max }\left(=\delta r_{\max }^{A}=\delta r_{\max }^{d}\right)$ is always associated with a particular permutation of the end-points of the coefficient intervals $\left[g_{n}^{m}-\delta g_{n}^{m}\right.$, $\left.g_{n}^{m}+\delta g_{n}^{m}\right]$ and $\left[h_{n}^{m}-\delta h_{n}^{m}, h_{n}^{m}+\delta h_{n}^{m}\right]$, as discussed in 


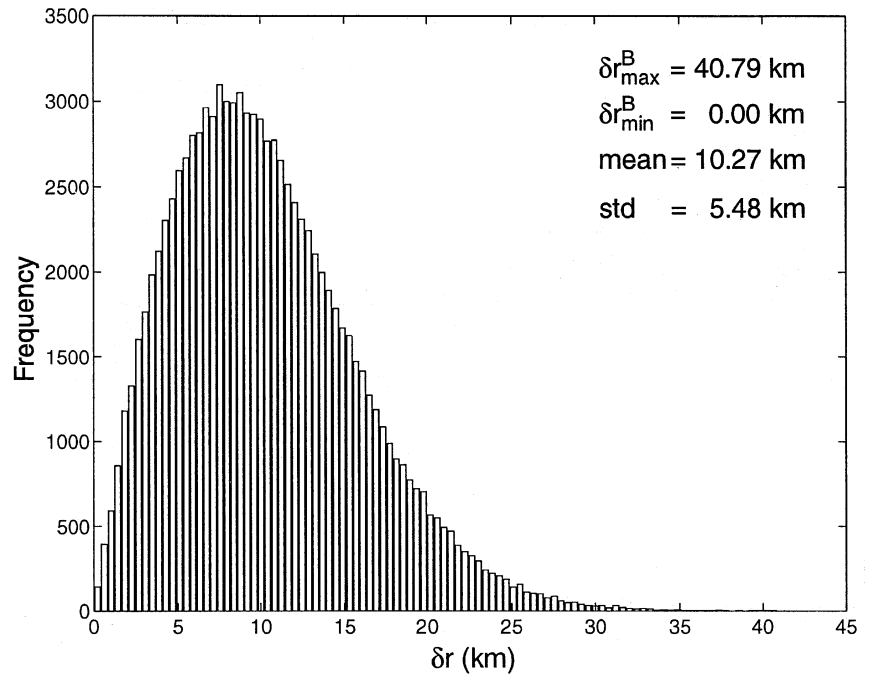

a

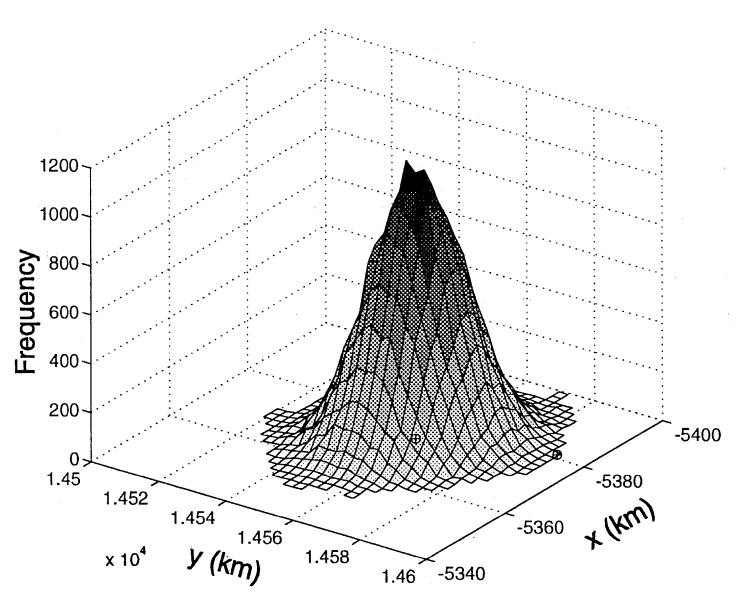

c

Isometric Projection

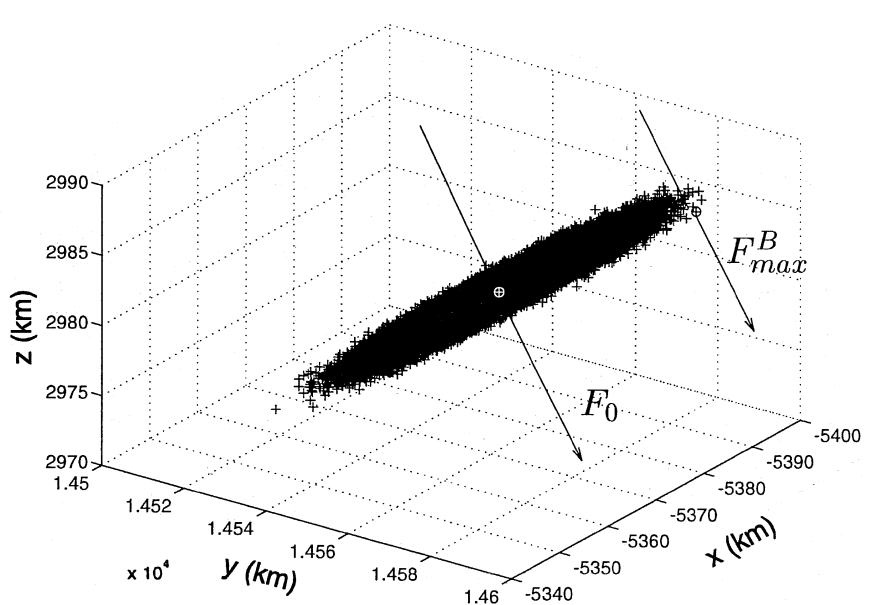

b

Scatter Plot
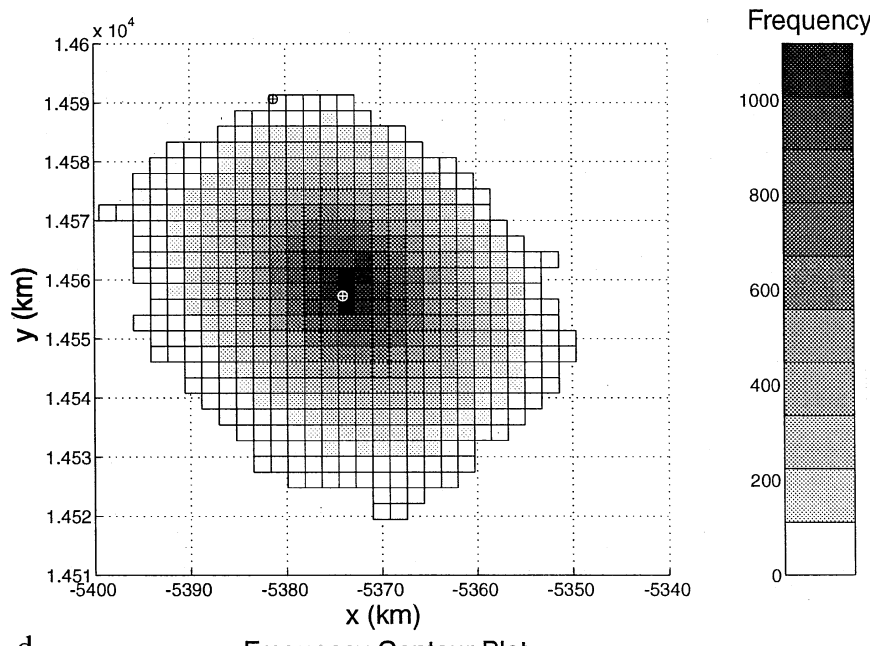

Fig. 3a-d. Same as Fig 1, but for Longitude 2 and $r_{1}=2 R_{\mathrm{E}}$

Sects. 4.1 and 4.2, internal points in these coefficient intervals do not yield values of $\delta r$ that lie outside the uncertainty interval $\left[0, \delta r_{\max }\right]$. Therefore, the validity of Conjecture 2 is established. It should be noted that all four histograms reach a maximum that is well displaced from the origin, contrary to the situation in Figs. 1 and 2 of Paper I. In the case of the complete (non-axisymmetric) internal geomagnetic field, the geomagnetic equatorial crossing points form a two-dimensional distribution and the class intervals are defined by annular areas. Hence the shape of each histogram is determined by a decreasing density of points with increasing radial distance from the origin combined with a corresponding increase in the areas of the class intervals.

The scatter plots in Figs. 1b-4b also show the orientation of the magnetic field line $\left(F_{0}\right)$ that passes through the central point $(\delta r=0)$ and the orientation of the magnetic field line $\left(F_{\max }^{B}\right)$ that passes through the furthest point $\left(\delta r_{\max }^{B}\right)$; the arrows merely signify the directions of the field-line tracings. The central and furthest points are both represented by the symbol $\oplus$ in panels b, c and d of Figs. 1-4: clearly, the central point $(\delta r=0)$ is near the centre of the distribution of geomagnetic equatorial crossing points and the furthest point is at the very edge of the distribution. Panels b, c and d of Figs. 1-4 illustrate the fact that the distribution of crossing points is elongated radially, rather than longitudinally, which is to be expected because magnetic field lines do not deviate drastically from dipolar geomagnetic meridian planes. Panels $\mathrm{c}$ and d in Figs. 1-4 should be interpreted with some circumspection, since they show projections from the geomagnetic equatorial plane defined in Sect. 4.3 onto the geographic equatorial plane.

\section{Uncertainties in the conjugate points of selected geophysical observatories}

Table 4 presents estimates of the maximum uncertainties in the locations of the conjugate points of selected geophysical observatories. This table gives the geographic coordinates of the geophysical observatories, which define the initial points of the field-line tracings, and the geographic coordinates of their conjugate 


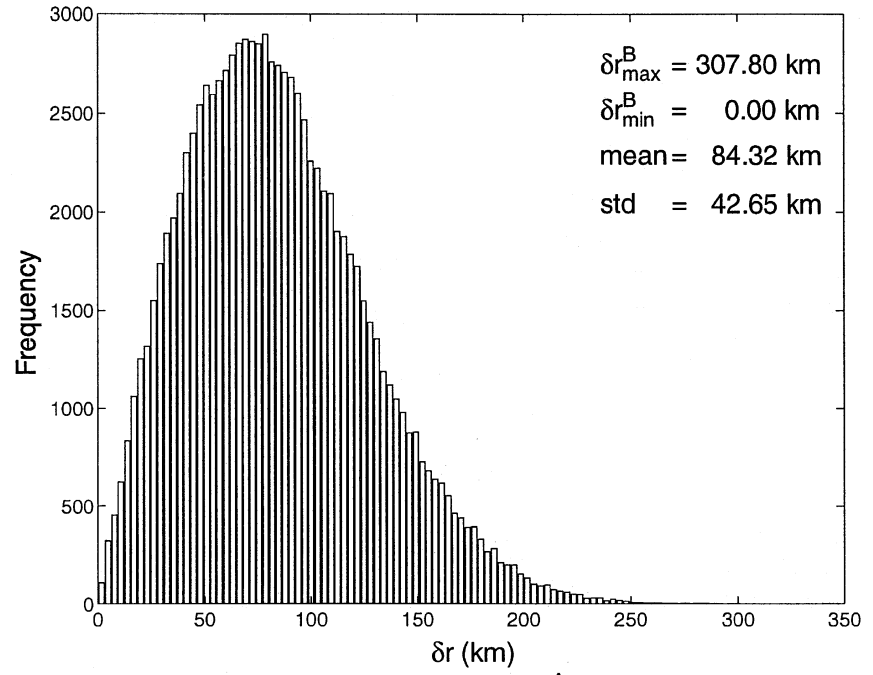

a

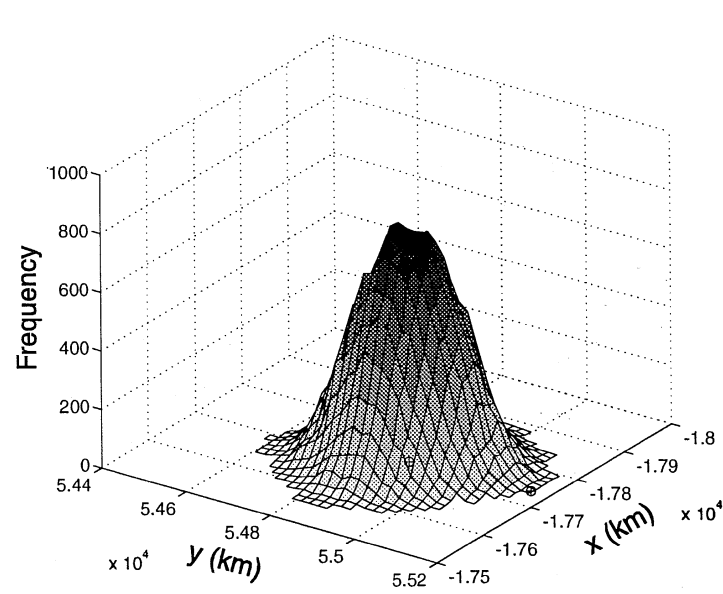

C

Isometric Projection

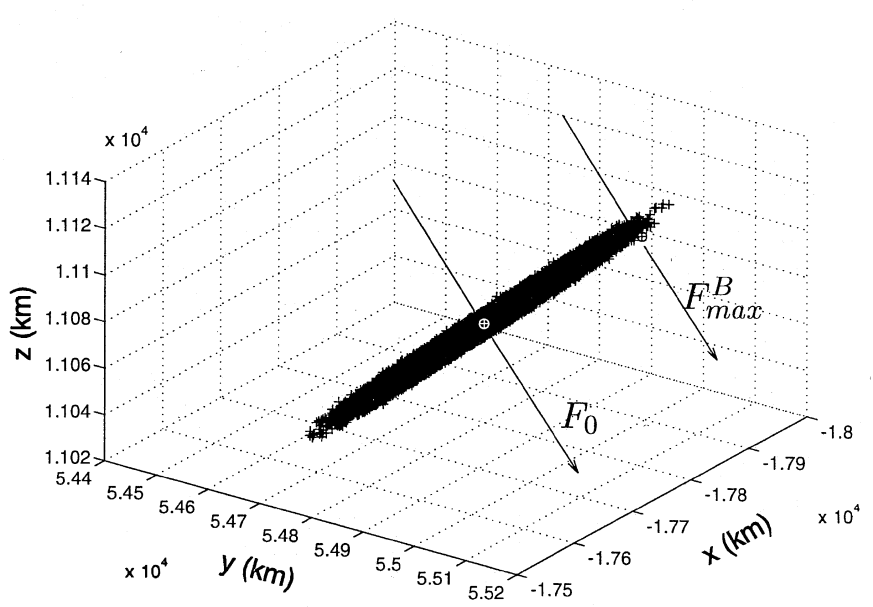

b
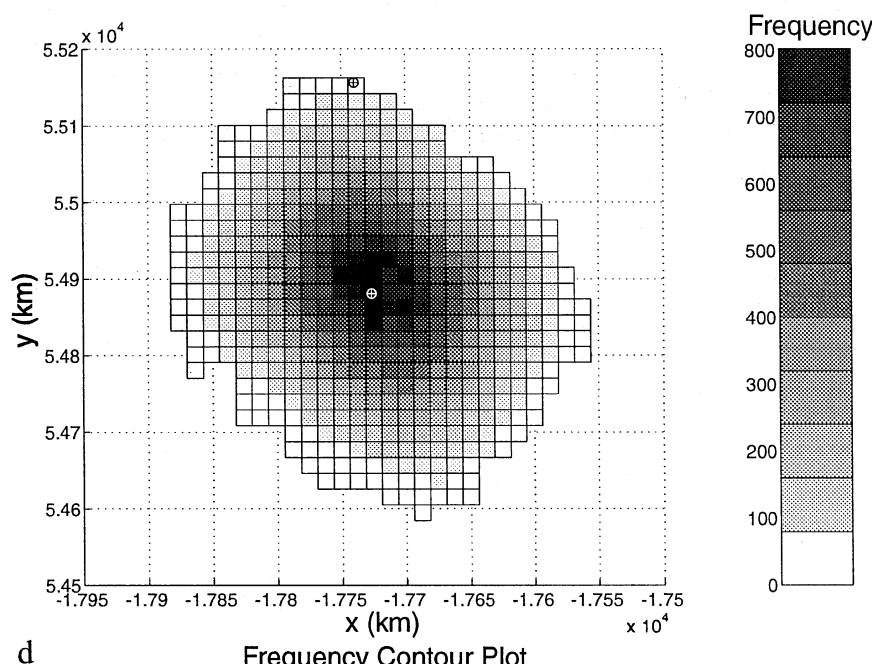

Fig. 4a-d. Same as Fig 1, but for Longitude 2 and $r_{1}=6 R_{\mathrm{E}}$

points, which are defined by the end-points of the fieldline tracings at the surface of the Earth in the opposite hemisphere. The field-line tracings are performed using the computer program described in Sect. 3 and the maximum uncertainties $\delta r_{\max }$ are calculated using the algorithmic procedure formulated in Sect. 4.1, so that $\delta r_{\max }=\delta r_{\max }^{A}=\delta r_{\max }^{d}$ with the nomenclature adopted in Sects. 4.1 and 5.1. Table 4 also gives the maximum uncertainties in the geomagnetic equatorial crossing points, which are defined in Sect. 4.3. The conjugate points and their associated uncertainties are calculated using the GSFC 1990D model of the internal geomagnetic field. The spherical harmonic coefficients of this model and their standard errors are listed in Appendix B. The values of $\delta r_{\max }\left(=\delta r_{\max }^{A}\right)$ at both the equator and the conjugate point are calculated assuming that each $\delta g_{n}^{m}$ and $\delta h_{n}^{m}$ is equal to the corresponding standard error in Appendix B. As noted in Sect. 4.3 (and Paper I), if each $\delta g_{n}^{m}$ and $\delta h_{n}^{m}$ is taken to be the same multiple (say $\lambda$ ) of the standard error, then the associated uncertainties can be obtained approximately by multiplying the values of $\delta r_{\max }$ in Table 4 by the factor $\lambda$. Therefore, with the assumptions made in this investigation, it can be concluded at the $3 \sigma$ confidence level of $99.73 \%$ (for the spherical harmonic coefficients) that upper limits on the uncertainties in the locations of the conjugate points are obtained approximately by tripling the values of $\delta r_{\max }$ in Table 4. Similarly for the geomagnetic equatorial crossing points. Conversely, assuming random errors $\Delta g_{n}^{m}$ and $\Delta h_{n}^{m}$ in the error intervals $\left[-\delta g_{n}^{m},+\delta g_{n}^{m}\right]$ and $\left[-\delta h_{n}^{m},+\delta h_{n}^{m}\right]$ yields values of $\delta r_{\max }^{B}$, calculated according to Method B in Sec. 5.1, which are about half those given in Table 4 for $\delta r_{\max }^{A}$. This latter conclusion is corroborated by the illustrative results presented in Figs. 1-4.

Certain properties of $\delta r_{\max }$ can be inferred from visual inspection of the numerical estimates presented in Table 4. The value of $\delta r_{\max }$ at the geomagnetic equator increases with increasing geographic latitude for starting positions in either the northern or southern hemisphere. Conversely, the value of $\delta r_{\max }$ at the conjugate point decreases with increasing geographic latitude. These 
results can be interpreted qualitatively in terms of fieldline tracing as follows. Two adjacent field lines at the same longitude diverge continuously with increasing distance from the Earth until the geomagnetic equatorial plane is reached and then they converge continuously until the conjugate points are reached. Moreover, for pairs of field lines that are equally separated in latitude at the surface of the Earth, the separation in the geomagnetic equatorial plane increases with increasing magnetic latitude, or increasing geocentric distance in the geomagnetic equator. Conversely, for pairs of field lines that are equally separated radially in the geomagnetic equatorial plane, the corresponding separation at the surface of the Earth decreases with increasing magnetic latitude, or increasing geocentric distance in the geomagnetic equator.

\section{Summary and conclusions}

As noted in the preceding paper by Willis, Singh and Comer (1997), which is referred to as Paper I, the goal of this investigation is to begin a systematic study of the uncertainties in magnetic-field-line tracing arising from uncertainties in the specification of the six main sources of magnetic field in the magnetosphere. These six sources are: (1) currents flowing in the Earth's liquid metallic outer core; (2) ionospheric currents; (3) fieldaligned (or Birkeland) currents; (4) ring currents; (5) magnetopause currents; and (6) magnetotail currents (Stern and Tsyganenko, 1992). Following the rationale adopted in Paper I, it seems logical to commence such a systematic study with a detailed examination of the uncertainties in field-line tracing in the magnetosphere produced solely by possible errors in the specification of the geomagnetic field of internal origin (Sect. 2). As in Paper I, the primary purpose of the present investigation is to estimate these uncertainties by using one of the few published models of the geomagnetic field that gives both the spherical harmonic coefficients and their standard errors (Langel et al., 1989, 1992). Because of the considerable complexities in computing these uncertainties in field-line tracing for the complete geomagnetic field of internal origin, attention is focused in Paper I on the uncertainties that result from the standard errors in just the axisymmetric part of the internal geomagnetic field. The results presented in
Paper I are generalized in this paper to the case of the complete internal geomagnetic field.

Since there is no known analytic equation for the magnetic field lines in the case of the complete (nonaxisymmetric) geomagnetic field of internal origin, uncertainties in field-line tracing in the magnetosphere are calculated using stepwise numerical integration along magnetic field lines. The computer program used to trace along magnetic field lines is discussed in detail, with particular emphasis on the actual procedures employed (Sect. 3.2). In the case of the axisymmetric part of the internal geomagnetic field, the maximum uncertainties in field-line tracing in the magnetosphere $\left(\delta r_{\max }\right)$ derived by stepwise numerical integration along magnetic field lines are essentially identical to those derived independently by the iterative numerical procedure for finding the roots of the polynomial equation derived by Backus (1988), as noted in Paper I (Sec. 5). The excellent agreement between results obtained by these two different techniques confirms that the computer program used for stepwise numerical integration is very accurate. However, evaluating uncertainties in field-line tracing in the magnetosphere by considering all permutations of the end-points of the uncertainty intervals for the spherical harmonic coefficients (i.e. $g_{n}^{m} \pm \delta g_{n}^{m}$ and $h_{n}^{m} \pm \delta h_{n}^{m}$ for $0 \leq m \leq n$ and $\left.1 \leq n \leq 10\right)$ is only practicable computationally in the case of the axisymmetric part $(m=0)$ of the internal geomagnetic field $\left(N=n_{\max }=10\right)$. For the complete internal geomagnetic field, the all-permutations approach is not really feasible beyond $n_{\max }=3(N=15)$ because the computing times are prohibitively long even on a Cray $\mathrm{Y}$ - MP computer (Appendix A).

An algorithm that drastically reduces the computing time required to estimate the uncertainties in field-line tracing in the magnetosphere has been formulated (Sect. 4.1). In the case of the complete internal geomagnetic field $\left(n_{\max }=10, N=120\right), 122$ field-line tracings are required in the algorithmic procedure, compared with $2^{120}+1$ field-line tracings in the permutational procedure (Sect. 4.2). This reduction in the number of fieldline tracings represents a quite remarkable curtailment of the necessary computing time. The agreement between the algorithmic and permutational procedures is compared and confirmed numerically in two special cases: (i) the axisymmetric part $(m=0)$ of the internal geomagnetic field in the general case $N=n_{\max }=10$, and

Table 4. Numerical estimates for $\delta r_{\max }$ at the geomagnetic equator and at the conjugate point for selected geophysical observatories

\begin{tabular}{|c|c|c|c|c|c|c|}
\hline \multirow{3}{*}{$\begin{array}{l}\text { Geophysical } \\
\text { Observatory }\end{array}$} & \multicolumn{2}{|c|}{ Site Coordinates } & \multicolumn{2}{|c|}{ Conjugate Point } & \multirow{3}{*}{$\begin{array}{l}\delta r_{\max } \\
\text { Geomagnetic } \\
\text { Equator }(\mathrm{km})\end{array}$} & \multirow{3}{*}{$\begin{array}{l}\delta r_{\max } \\
\text { Conjugate } \\
\text { Point }(\mathrm{km})\end{array}$} \\
\hline & Geographic & Geographic & Geographic & Geographic & & \\
\hline & Latitude $\left({ }^{\circ} \mathrm{N}\right)$ & Longitude $\left({ }^{\circ} \mathrm{E}\right)$ & Latitude $\left({ }^{\circ} \mathrm{N}\right)$ & Longitude $\left({ }^{\circ} \mathrm{E}\right)$ & & \\
\hline Longyear byen & 78.15 & 16.03 & -66.29 & 85.01 & 1385.14 & 20.76 \\
\hline Tromsø & 69.58 & 19.22 & -60.85 & 65.55 & 419.67 & 26.42 \\
\hline Goose Bay & 53.32 & 299.64 & -77.37 & -38.29 & 280.77 & 30.63 \\
\hline South Georgia & -54.28 & 323.52 & 35.50 & -56.61 & 108.06 & 45.43 \\
\hline Argentine Islands & -65.25 & 295.73 & 38.93 & -68.84 & 137.88 & 35.08 \\
\hline Halley & -75.58 & 333.63 & 53.52 & -55.93 & 280.56 & 27.27 \\
\hline South Pole & -90.00 & 0.00 & 64.54 & -66.46 & 1046.37 & 21.12 \\
\hline
\end{tabular}


(ii) the complete $(m \neq 0)$ internal geomagnetic field in the restrictive case $n_{\max }=3, N=15$. On the basis of these two successful comparisons, it is assumed that the algorithmic procedure can be used with confidence in those cases for which the permutational procedure involves inordinately long computing times.

Tables 2 and 3 provide numerical estimates of the maximum uncertainties, $\delta r_{\max }$, in the geocentric distance at which a magnetic field line crosses the geomagnetic equatorial plane for the complete geomagnetic field of internal origin. As in Paper I, results are presented for nominal (dipolar) magnetic equatorial crossing distances of $r_{1}=2$ and $6 R_{\mathrm{E}}$. Since the complete internal geomagnetic field is non-axisymmetric, the uncertainties in field-line tracing in the magnetosphere depend on both the co-latitude $(\theta)$ and longitude $(\phi)$ of the starting point (Sect. 4.3). All numerical estimates of the maximum uncertainty $\left(\delta r_{\max }\right)$ in field-line tracing in the magnetosphere are based on the NASA GSFC 1990D model of the internal geomagnetic field (Appendix B) and are derived using the algorithmic procedure (Sect. 4.1). Tables 2 and 3 give illustrative numerical values for $\delta r_{\max }$ in the two special geographic longitudes defined by the centred geomagnetic dipole axis (i.e. defined by $g_{1}^{0}, g_{1}^{1}$ and $h_{1}^{1}$ ). For the complete internal geomagnetic field $\left(n_{\max }=10, N=120\right)$, the maximum characteristic uncertainty in the geocentric distance of a field line that crosses the geomagnetic equator at a nominal dipolar distance of $2 R_{\mathrm{E}}$ is typically about $100 \mathrm{~km}$ (Tables 2 and 3 ). The corresponding characteristic uncertainty for a field line that crosses the geomagnetic equator at a nominal dipolar distance of $6 R_{\mathrm{E}}$ is typically about 500 $\mathrm{km}$ (Tables 2 and 3). These values are substantially larger than those for the axisymmetric part of the internal geomagnetic field $\left(N=n_{\max }=10\right)$, namely 10 and $70 \mathrm{~km}$ (Table 1). The increases in $\delta r_{\max }$ are a direct consequence of the fact that there are 120 standard errors for the complete geomagnetic field, compared with only 10 for the axisymmetric part.

Table 4 presents estimates of the maximum uncertainties $\left(\delta r_{\max }\right)$ in the locations of the conjugate points of selected geophysical observatories. This table gives the geographic coordinates of these observatories and their conjugate points in the opposite hemisphere, which are determined by field-line tracing. All field-line tracings are accomplished using the computer program that performs stepwise numerical integration along magnetic field lines (Sect. 3.2) and the maximum uncertainties $\left(\delta r_{\max }\right)$ are again calculated using the algorithmic procedure (Sect. 4.1). For completeness, Table 4 also gives the maximum uncertainties of the geomagnetic equatorial crossing points (Sect. 4.3). It can be inferred from visual inspection of the numerical estimates presented in Table 4 that the value of $\delta r_{\max }$ at the geomagnetic equator increases with increasing geographic (geomagnetic) latitude of the observatory. Conversely, the value of $\delta r_{\max }$ at the conjugate point decreases with increasing geographic (geomagnetic) latitude of the observatory. Both these conclusions are easily explained in terms of the configuration of the magnetic field lines of a predominantly dipolar internal geomagnetic field (Sect. 6).

Numerical estimates of the uncertainties in magneticfield-line tracing in the magnetosphere, which are calculated in this paper for the complete internal geomagnetic field, should be regarded as "first approximations", in the sense that such estimates are only as accurate as the published standard errors in the full set of spherical harmonic coefficients (Langel et al., 1989, 1992). Once again, however, all the procedures developed in this paper can be applied to the derivation of more realistic estimates of the uncertainties in magneticfield-line tracing in the magnetosphere, following further progress in the determination of more accurate standard errors in the spherical harmonic coefficients.

Finally, it must be emphasized again that the results presented in this paper and in Paper I apply strictly to the geomagnetic field of internal origin. Further research is required to determine accurately the uncertainties in field-line tracing arising from the presence of external sources of magnetic field in the magnetosphere; for example, ionospheric currents, field-aligned (or Birkeland) currents, ring currents, magnetopause currents and magnetotail currents.

Acknowledgements. The authors are greatly indebted to Dr D.R. Barraclough, Dr M.A. Hapgood and Dr A.S. Rodger for valuable advice and thank Mr S.R. Crothers, Mr V.N. Davda and Mrs J. Foster for considerable help with the preparation of the paper. The research reported in this paper was undertaken while JRS pursued a twelve-month period of Professional Training at the Rutherford Appleton Laboratory as part of the BSc. Honours Degree Course in Mathematics at Coventry University and continued this research as a fourth-year project.

Topical Editor K.-H. Glaßmeier thanks D.R. Barraclough, G. Backus and another referee for their help in evaluating this paper.

\section{Appendix A. Catalogue of cases considered and estimates of computing times required}

The following catalogue lists the different cases considered and provides characteristic estimates of the computing times required to perform the corresponding calculations. All the various computational methods considered in Paper I $\left[N=n_{\max }\right]$ and in this paper $\left[N=n_{\max }\left(n_{\max }+2\right)\right]$ are included. "Polynomials" refers to numerical solutions of the polynomial equation defined and discussed in Sects. 4.3 and 5 of Paper I. "Tracings" refers to numerical results obtained using the computer program that traces along magnetic field lines, as discussed in Sect. 3 of this paper. The symbol $\checkmark$ signifies cases for which the computations have been performed on a DEC 3000 AXP (Alpha) - Model 400 computer and thus the computing times are known from practical experience. The symbol $\boldsymbol{X}$ signifies cases for which the computations have not been performed because estimates of the computing times are inordinately long even on a Cray Y - MP computer.

$$
\begin{gathered}
{\left[\boldsymbol{N}=\boldsymbol{n}_{\max }\right]} \\
2^{10} \text { Polynomials } \approx 10 \mathrm{~s} \checkmark \\
\left(\text { all permutations } \pm \delta g_{n}^{0} ; N=10\right) \\
2^{10} \text { Polynomials } \approx 10 \mathrm{~s} \checkmark
\end{gathered}
$$

(10 random numbers $-\delta g_{n}^{0} \leq \Delta g_{n}^{0} \leq+\delta g_{n}^{0} ; N=10$ ) 


$$
\begin{aligned}
& 2^{10} \text { Tracings } \approx 1^{1 / 2} \mathrm{~h} \mathcal{V} \\
& \text { (all permutations } \pm \delta g_{n}^{0} ; N=10 \text { ) } \\
& 5^{10} \text { Polynomials } \approx 10 \mathrm{~h} \checkmark \\
& \text { (all permutations } 0, \pm(1 / 2) \delta g_{n}^{0}, \pm \delta g_{n}^{0} ; N=10 \text { ) } \\
& 5^{10} \text { Polynomials } \approx 10 \mathrm{~h} \checkmark \\
& \text { (10 random numbers }-\delta g_{n}^{0} \leq \Delta g_{n}^{0} \leq+\delta g_{n}^{0} ; N=10 \text { ) } \\
& 5^{10} \text { Tracings } \approx 20 \text { days } X \\
& \text { (all permutations } 0, \pm(1 / 2) \delta g_{n}^{0}, \pm \delta g_{n}^{0} ; N=10 \text { ) } \\
& {\left[\boldsymbol{N}=\boldsymbol{n}_{\text {max }}\left(\boldsymbol{n}_{\max }+2\right)\right]} \\
& 2^{15} \text { Tracings } \approx 6 \mathrm{~h} \checkmark \\
& \text { (all permutations } \pm \delta g_{n}^{m}, \pm \delta h_{n}^{m} ; n_{\max }=3, N=15 \text { ) } \\
& 10^{5} \text { Tracings } \approx 8 \mathrm{~h} \checkmark \\
& \text { (120 random numbers }-\delta g_{n}^{m} \leq \Delta g_{n}^{m} \leq+\delta g_{n}^{m} \text {, } \\
& -\delta h_{n}^{m} \leq \Delta h_{n}^{m} \leq+\delta h_{n}^{m} ; n_{\max }=10, N=120 \text { ) } \\
& 2^{24} \text { Tracings } \approx 1 \text { month } \boldsymbol{X} \\
& \text { (all permutations } \pm \delta g_{n}^{m}, \pm \delta h_{n}^{m} ; n_{\max }=4, N=24 \text { ) } \\
& 2^{35} \text { Tracings } \approx 10^{2} \text { years } \boldsymbol{X} \\
& \text { (all permutations } \pm \delta g_{n}^{m}, \pm \delta h_{n}^{m} ; n_{\max }=5, N=35 \text { ) } \\
& 2^{120} \text { Tracings } \approx 10^{28} \text { years } \boldsymbol{X} \\
& \text { (all permutations } \pm \delta g_{n}^{m}, \pm \delta h_{n}^{m} ; n_{\max }=10, N=120 \text { ) }
\end{aligned}
$$

\begin{tabular}{|c|c|c|c|c|c|}
\hline 6 & 2 & 61.4 & 3.69 & 83.8 & 3.60 \\
\hline 6 & 3 & -174.8 & 3.69 & 68.6 & 3.78 \\
\hline 6 & 4 & 4.7 & 3.87 & -50.1 & 3.80 \\
\hline 6 & 5 & 17.4 & 3.51 & 3.8 & 3.69 \\
\hline 6 & 6 & -97.5 & 5.04 & 27.7 & 5.14 \\
\hline 7 & 0 & 74.1 & 2.11 & 0.0 & 0.00 \\
\hline 7 & 1 & -64.3 & 2.26 & -84.9 & 1.86 \\
\hline 7 & 2 & 4.7 & 2.24 & -27.8 & 2.03 \\
\hline 7 & 3 & 26.5 & 2.13 & 1.5 & 2.03 \\
\hline 7 & 4 & -1.0 & 2.21 & 22.2 & 2.22 \\
\hline 7 & 5 & 5.8 & 2.14 & 14.6 & 2.41 \\
\hline 7 & 6 & 9.8 & 2.05 & -23.7 & 2.21 \\
\hline 7 & 7 & -2.8 & 2.65 & -3.6 & 2.76 \\
\hline 8 & 0 & 22.3 & 1.84 & 0.0 & 0.00 \\
\hline 8 & 1 & 7.1 & 1.96 & 7.1 & 1.67 \\
\hline 8 & 2 & -0.2 & 1.90 & -19.9 & 1.84 \\
\hline 8 & 3 & -10.1 & 1.93 & 5.3 & 1.81 \\
\hline 8 & 4 & -10.0 & 1.99 & -23.7 & 1.97 \\
\hline 8 & 5 & 3.5 & 1.92 & 11.4 & 2.10 \\
\hline 8 & 6 & 3.6 & 1.98 & 12.5 & 2.18 \\
\hline 8 & 7 & 3.6 & 1.94 & -14.7 & 2.02 \\
\hline 8 & 8 & -4.5 & 2.51 & -12.9 & 2.56 \\
\hline 9 & 0 & 4.4 & 1.49 & 0.0 & 0.00 \\
\hline 9 & 1 & 10.2 & 1.61 & -20.1 & 1.55 \\
\hline 9 & 2 & 1.5 & 1.61 & 15.3 & 1.56 \\
\hline 9 & 3 & -12.7 & 1.62 & 10.0 & 1.67 \\
\hline 9 & 4 & 8.9 & 1.73 & -5.9 & 1.67 \\
\hline 9 & 5 & -5.0 & 1.73 & -6.7 & 1.88 \\
\hline 9 & 6 & -1.8 & 1.77 & 7.8 & 1.88 \\
\hline 9 & 7 & 8.1 & 1.84 & 8.4 & 1.89 \\
\hline 9 & 8 & 1.5 & 1.87 & -6.5 & 1.92 \\
\hline 9 & 9 & -5.9 & 2.40 & 1.7 & 2.36 \\
\hline 10 & 0 & -3.4 & 1.25 & 0.0 & 0.00 \\
\hline 10 & 1 & -4.4 & 1.25 & 0.9 & 1.21 \\
\hline 10 & 2 & 1.7 & 1.30 & 0.4 & 1.26 \\
\hline 10 & 3 & -5.5 & 1.32 & 3.5 & 1.27 \\
\hline 10 & 4 & -2.1 & 1.39 & 5.2 & 1.31 \\
\hline 10 & 5 & 4.0 & 1.43 & -4.4 & 1.42 \\
\hline 10 & 6 & 2.9 & 1.49 & -1.0 & 1.48 \\
\hline 10 & 7 & 1.1 & 1.54 & -1.8 & 1.53 \\
\hline 10 & 8 & 1.9 & 1.63 & 3.5 & 1.57 \\
\hline 10 & 9 & 2.4 & 1.77 & -0.8 & 1.78 \\
\hline 10 & 10 & -0.3 & 2.25 & -6.3 & 2.23 \\
\hline
\end{tabular}

\section{Appendix B. The GSFC 1990D Model}

The following table presents the spherical harmonic coefficients $\left(g_{n}{ }^{m}, h_{n}{ }^{m}\right)$ and their corresponding standard errors $\left(\delta g_{n}{ }^{m}, \delta h_{n}{ }^{m}\right)$ for the NASA Goddard Space Flight Center model of the internal geomagnetic field, designated GSFC 1990D (after Langel et al., 1992).

\begin{tabular}{llrrrr}
\hline$n$ & $m$ & \multicolumn{1}{l}{$\begin{array}{l}g_{n}^{m} \\
(\mathrm{nT})\end{array}$} & $\begin{array}{l}\delta g_{n}^{m} \\
(\mathrm{nT})\end{array}$ & \multicolumn{1}{l}{$\begin{array}{l}h_{n}^{m} \\
(\mathrm{nT})\end{array}$} & \multicolumn{1}{c}{$\begin{array}{l}\delta h_{n}^{m} \\
(\mathrm{nT})\end{array}$} \\
\hline 1 & 0 & -29771.0 & 10.06 & 0.0 & 0.00 \\
1 & 1 & -1851.2 & 12.42 & 5410.4 & 11.10 \\
2 & 0 & -2137.0 & 8.62 & 0.0 & 0.00 \\
2 & 1 & 3056.4 & 8.65 & -2276.7 & 7.74 \\
2 & 2 & 1695.1 & 9.71 & -380.2 & 10.51 \\
3 & 0 & 1313.5 & 8.21 & 0.0 & 0.00 \\
3 & 1 & -2235.8 & 8.37 & -283.6 & 7.55 \\
3 & 2 & 1243.8 & 6.94 & 293.0 & 6.88 \\
3 & 3 & 810.8 & 9.24 & -346.4 & 9.98 \\
4 & 0 & 939.1 & 6.70 & 0.0 & 0.00 \\
4 & 1 & 779.6 & 7.11 & 245.6 & 5.89 \\
4 & 2 & 323.9 & 6.85 & -242.3 & 7.00 \\
4 & 3 & -423.3 & 5.71 & 86.5 & 5.69 \\
4 & 4 & 138.2 & 9.30 & -299.4 & 9.01 \\
5 & 0 & -211.0 & 4.28 & 0.0 & 0.00 \\
5 & 1 & 351.9 & 4.50 & 49.6 & 3.90 \\
5 & 2 & 241.8 & 4.41 & 154.7 & 4.29 \\
5 & 3 & -112.2 & 4.62 & -156.2 & 4.56 \\
5 & 4 & -168.4 & 3.90 & -71.1 & 4.04 \\
5 & 5 & -38.7 & 5.53 & 98.9 & 5.73 \\
6 & 0 & 60.8 & 3.45 & 0.0 & 0.00 \\
6 & 1 & 63.9 & 3.85 & -15.7 & 3.36 \\
\hline
\end{tabular}

\section{References}

Backus, G. E., The field lines of an axisymmetric magnetic field, Geophys. J., 93, 413-417, 1988.

Jeffreys, B., Derivations of the equation for the field lines of an axisymmetric multipole, Geophys. J., 92, 355-356, 1988.

Kluge, G., A generalised method for the calculation of the geomagnetic field from multipole expansions, ESOC Internal Note No. 61, Darmstadt, Germany, 1970a.

Kluge, G., Calculation of field lines and the shell parameter L from multipole expansions of the geomagnetic field, ESOC Internal Note No. 66, Darmstadt, Germany, 1970b.

Kluge, G., Computer program SHELL for the calculation of B and $\mathrm{L}$ from models of the geomagnetic field, ESOC Internal Note No. 67, Darmstadt, Germany, 1970c.

Kluge G., Direct computation of the magnetic shell parameter, Comput. Phys. Commun., 3, 31-35, 1972.

Langel, R. A., International Geomagnetic Reference Field, 1991 revision, Pure Appl. Geophys., 137, 301-308, 1991.

Langel, R. A., International Geomagnetic Reference Field: the sixth generation, J. Geomagn. Geoelectr.,44, 679 - 707, 1992.

Langel, R. A., R. H. Estes, and T. J. Sabaka, Uncertainty estimates in geomagnetic field modeling, J. Geophys. Res., 94, 12281$12299,1989$. 
Langel, R. A., T. J. Sabaka, and R. T. Baldwin, The geomagnetic field: 1970-1990 and the NASA candidate models for DGRF 1985 and IGRF 1990, J. Geomagn. Geoelectr., 44, 745-767, 1992.

Malin, S. R. C., and D. R. Barraclough, An algorithm for synthesizing the geomagnetic field, Comput. Geosci., 7, 401405, 1981.

MATLAB Reference Guide, The Math Works, Natick, Mass., 1992.

Stern, D. P., and N. A. Tsyganenko, Uses and limitations of the Tsyganenko magnetic field models, EOS Trans. Am. Geophys. Union, 73, 489, 493 - 494, 1992.
Willis, D. M., and A. R. Gardiner, Equations for the field lines of a sectorial magnetic multipole, Geophys. J., 95, 625-632, 1988.

Willis, D. M., and L. R. Young, Equation for the field lines of an axisymmetric magnetic multipole, Geophys. J.R. Astron. Soc., 89, 1011-1022, 1987.

Willis, D. M., J. R. Singh, and J. Comer, Uncertainties in field-line tracing in the magnetosphere. Part I: the axisymmetric part of the internal geomagnetic field, Ann. Geophysicae, submitted, 1997. 